\title{
Ist Standarddeutsch für Deutschschweizer eine Fremdsprache? Untersuchungen zu einem Topos des sprachreflexiven Diskurses
}

\author{
Sara Hägi (Bonn)/Joachim Scharloth (Zürich)
}

\begin{abstract}
This paper is concerned with the question, whether the status of Standard German in Germanspeaking Switzerland is adequately described as that of a foreign language. It discusses typological aspects, language awareness and language ideologies among German-speaking Swiss people, the practice of language acquisition, the language use in private life and media and the linguistic discourse about the relationship between the use of Swiss German and Standard German. It argues that from a linguistic point of view in none of these fields a clear decision can be made whether Standard German is a foreign language or not. Thus, the authors suggest that the conceptual framework ought to be widened to adequately describe the status of Standard German in German-speaking Switzerland. Finally, they take occasion to develop the concept of "Sekundärsprache"/"secondary language" for language situations similar to that in German-speaking Switzerland.
\end{abstract}

\section{$1 \quad$ Fragestellung und Relevanz}

"Fallt's Ihne schwär, innere Fremdsprach z'schrybe?". "Sicher", sagte ich. Kurz zuvor hatte ich mich in der Lage gefunden, Vorlesungen auf englisch halten zu müssen [...]. Aber die Lehrerin hatte mit ihrer Frage "hochdeutsch" im Visier. Da wollte ich wissen, ob für sie Gottfried Keller und Robert Walser fremdsprachige Autoren seien. "Ich ha nu gmeint", antwortete sie. Das hatte auch ich. (Loetscher 1986: 24)

"[D]e facto ist für Schweizerdeutsche Kinder das ab der ersten Klasse unterrichtete Hochdeutsch bereits die erste Fremdsprache"1 - Aussagen dieser Art liest und hört man immer wieder $^{2}$ und häufig ist damit eine Erklärung oder gar Entschuldigung für allfällige Fehler, Versprecher oder Unsicherheiten verbunden. Die Kompetenz, richtig Hochdeutsch zu können, die liegt bei den Deutschen, so die gängige Meinung. Entsprechend sprachlich unterlegen fühlt man sich ihnen, und zwar noch bevor man mit einer gutmütig lächelnden Reaktion von einem deutschen Kommunikationspartner konfrontiert wurde - und noch lange nach einer solchen. Handelt es sich dabei nun einfach um einen letztlich eingebildeten "Hochdeutsch-

\footnotetext{
${ }^{1}$ Stichwort "Fremdsprache", Wikipedia. http://de.wikipedia.org/wiki/Fremdsprache (26.08.2004).

2 Cf. dazu auch Haas (2004: 94), der in diesem Zusammenhang auf Sieber/Sitta (1986: 29ff.), Häcki Buhofer/Burger (1998: 15f.), aber auch auf Schläpfer/Gutzwiller/Schmid (1991: 130) verweist, oder Berthele (2004: 127ff.).
} 
komplex", um Hemmungen, die sich nicht nur erklären, sondern auch überwinden lassen, oder können die Deutschen tatsächlich besser Deutsch? Und zwar einfach aus dem Grund, dass es für sie die Muttersprache ist, für Deutschschweizer hingegen eine Fremdsprache? Liegt es also in der Natur der Sache, dass Texte eines deutschen Linguisten von seinen Deutschschweizer Kolleginnen und Kollegen sprachlich nur sehr vorsichtig korrigiert ("höchstens unterschlängelt") werden, da man ihm diesbezüglich einfach die größere Kompetenz einräumt? Und müssen Deutschschweizer Autoren in der Regel von Deutschen lektoriert werden, auch wenn sie nicht in deutschen Verlagen publizieren?

Warum sind es in der Regel Deutsche, die Deutschschweizer Fernsehmoderatoren sprachlich schulen? Auffällig ist auch die Zahl Deutscher, die in der Deutschschweiz im Bereich Deutsch als Fremdsprache tätig sind: Vor allem auf dem wirtschaftlichen Sektor sind ihre Chancen deutlich besser als die ihrer deutschschweizerischen Kolleginnen und Kollegen. Es zeigt sich also durchgängig immer wieder das gleiche Bild, das nicht zuletzt, wenn auch polemisch und wohl mit anderen Hintergedanken in Bezug auf die Primarschule erst kürzlich wieder diskutiert wurde, als ein Abgeordneter der SVP den Vorschlag machte, "massenhaft Lehrer aus Deutschland zu holen. Die seien sehr viel billiger und würden wohl zumindest im Fach Deutsch die Kinder zu besseren Leistungen führen" (Altwegg 2004): Eigentlich können die Schweizer halt nicht richtig Deutsch.

Fragt man jedoch einen Deutschschweizer konkret, analog zu Loetscher im obigen Zitat, "und für dich, ist Hochdeutsch für dich etwa auch eine Fremdsprache?", so erhält man eher eine ausweichende Antwort. Bei entsprechenden Umfragen ${ }^{3}$ ist allerdings zu bedenken, dass das Wort Hochdeutsch in der Schweiz vielleicht eher mit dem deutschländischen Standard und weniger mit der schweizerischen Varietät assoziiert wird. Würde man nach den Einstellungen zu Schriftdeutsch gefragt, sähen die Antworten wahrscheinlich anders aus.

Über eines scheint man sich einig zu sein: Die Sprachsituation der deutschen Schweiz hat etwas Auffälliges an sich. Auch was diese Auffälligkeit ausmacht, ist seit rund zweihundertfünfzig Jahren kaum bestritten: Es ist der allgemeine Gebrauch von "Dialekten" in der alltäglichen Kommunikation, damit das Fehlen einheimischer Gruppen, die sich in dieser Situation der "Hochsprache" bedienen. (Haas 2004: 81)

Dies hat natürlich entsprechende Konsequenzen für die Verwendung und den Umgang mit den Varietäten, aber auch für deren Wahrnehmung und Wirkung.

In folgendem Ausschnitt aus dem Roman Bis bald des Deutschschweizer Schriftstellers Markus Werner (S. 38f) wird Hochdeutsch eindeutig als Fremdsprache verstanden:

Anfangs ist es mir störend vorgekommen, dass Regina - natürlich konnte sie nicht anders - mit unserem Säugling schriftdeutsch sprach, hamburgisch [...]. Übrigens habe ich unsere unterschiedliche Sprache schon vorher oft als etwas Trennendes erlebt. Gerade Herzenssachen erwiesen sich für mich, sobald ich sie formulieren wollte, als muttersprachliche Herzenssachen, und

\footnotetext{
3 So haben z. B. in einer kleinen (wohl nicht standardisierten) Umfrage von Berthele (2004: 127) 11 von 20 Befragten "auf die Frage, was sie für eine Beziehung zum Hochdeutschen hätten, spontan mit dem Begriff 'Fremdsprache' geantwortet, 7 haben auf Nachfrage, ob Hochdeutsch für sie eine Fremdsprache sei, mit 'Ja' geantwortet, und zwei der Befragten empfanden Hochdeutsch nicht als eine Fremdsprache."
} 
je heißer das Empfinden war, desto kühler tönte die Übersetzung. Es ist uns Schweizern, zumindest im mündlichen Verkehr und abgesehen von der Furcht vor Kasusfehlern, kaum möglich, auf hochdeutsch Liebe oder Leidenschaft zu äußern, ohne uns künstlich zu finden. Und umgekehrt, uns klingen Liebesworte, hochdeutsch in unser Ohr gestammelt, ein wenig unvertraut, ja fast gestelzt, so stammelt man sonst nur am Bildschirm und im Schauspielhaus, kurzum, es hat mir immer weh getan, daß Fremdheit ausgerechnet im intimen Bereich spürbar wurde.

Inwiefern das verbreitete Ideologem, Hochdeutsch sei des Deutschschweizers erste Fremdsprache, gerechtfertigt und wissenschaftlich haltbar ist, soll im Folgenden untersucht werden. Interessant ist die Fragestellung nicht nur in Hinblick auf die Einstellung der Deutschschweizer zur Standardsprache, sondern vor allem in Bezug auf die Konsequenzen für den muttersprachlichen wie für den Deutsch-als-Fremd- bzw. -Zweitsprache-Unterricht (DaF bzw. DaZ)4: Wäre Standarddeutsch für Deutschschweizer tatsächlich eine Fremdsprache, müsste es einerseits in der Schule wie an der Universität entsprechend unterrichtet werden. Andererseits müsste die Schweiz als auch deutschsprachiges Land ihre Aufgaben und Möglichkeiten im DaF-/DaZ-Bereich neu überdenken, nicht zuletzt ihre Attraktivität für Sprachaufenthalte. Doch nicht nur für den Bereich Deutsch als Fremd- bzw. Zweitsprache hätte eine Kategorisierung des Standarddeutschen als Fremdsprache Konsequenzen: Etwa müsste die Konstruktion einer schweizerischen Norm des Standarddeutschen, wie sie seit einigen Jahren von der Linguistik forciert wird, überdacht werden. Auch der Status der Mundart, die dann die einzige Muttersprache wäre, müsste in Schule, Verwaltung und Medien verbessert und ihr Ausbau zur Schriftsprache zumindest erwogen werden.

Die Sprachwissenschaft hat sich immer wieder mit der Frage beschäftigt, ob Standarddeutsch für Schweizer nun eine Fremdsprache sei oder nicht. Die Frage wurde in unterschiedlichen Zusammenhängen diskutiert und je nach Problemstellung anders formuliert. So lautet die Frage im schweizerisch-sprachpatriotischen Kontext (Baur 1983): Sind die in der Schweiz gesprochenen alemannischen Dialekte Varietäten der historischen Gesamtsprache Deutsch oder eigenständige Sprachen? Oder, um die sprachpatriotische Komponente deutlicher hervortreten zu lassen: Ist "Schweizerdeutsch" eine eigenständige Sprache? Wird diese Frage mit "ja" beantwortet, ist für die Autoren im Umkehrschluss Hochdeutsch eine Fremdsprache. Immer wieder thematisiert wurde dieser Fremdsprachencharakter des Standarddeutschen im Kontext des Problemkreises Dialekt und Schule (Sieber/Sitta 1986; Sieber 1990; Schmidlin 1999) sowie in Untersuchungen $\mathrm{zu}$ den Einstellungen der Deutschschweizer zum Standarddeutschen (Scharloth 2003). Die Auseinandersetzung mit dem argumentativen Topos, demzufolge Hochdeutsch für Deutschschweizer die erste Fremdsprache sei, wird hier als Faktor im Erwerb des Standarddeutschen in der Schule und als Ursache für Gebrauchsunsicherheit und -hemmungen beschrieben.

\footnotetext{
4 Deutsch als Zweitsprache bezeichnet, im Unterschied zu Deutsch als Fremdsprache im engeren Sinne, Erwerb, Vermittlung und den alltäglichen Gebrauch der deutschen Sprache innerhalb des deutschen Sprachraums (cf. dazu Götze u. a. 2003: 521 und Barkowski 2003: 525 sowie Kap. 6.1).
} 
Ein besonderes Augenmerk wird der Frage nach dem Fremdsprachencharakter des Standarddeutschen im wissenschaftlichen Diskurs auch dort zuteil, wo die Sprachensituation in der Schweiz, insbesondere der Deutschschweiz, thematisiert wird (Ammon 1995; Haas 1988, 2004, I; Werlen 1998; Berthele 2004). In eine soziolinguistische Terminologie übersetzt lautet dann die Frage: Ist die Sprachensituation in der Deutschschweiz als Bilingualismus oder Diglossie zu charakterisieren? Für die Vertreter der Diglossie-Position sind die in der Deutschschweiz gesprochenen Dialekte ebenso wie die deutsche Standardsprache Varietäten des Deutschen und damit keine Fremdsprache(n). Für die Verfechter der BilingualismusHypothese hingegen handelt es sich beim Schweizer Alemannisch auf der einen Seite und dem Standarddeutschen auf der anderen um unterschiedliche Sprachen. Wer sind nun die Vertreter der unterschiedlichen Positionen und mit welchen Argumenten begründen sie ihre Entscheidung?

Dafür, dem Standarddeutschen die Stellung einer Fremdsprache zuzuerkennen, plädiert z. B. Arthur Baur (1983, 1990). Als Argumente für seine Position führt er zum einen die Ausgebautheit des Schweizerdeutschen und zum anderen den großen Abstand zwischen Standarddeutsch und den Dialekten ins Feld (Baur 1983: 37-41, 64f.). Demnach unterscheiden sich Standarddeutsch und die in der Schweiz gesprochenen alemannischen Dialekte in Lautung, Grammatik und Lexik so stark, dass es gerechtfertigt ist, sie als eigenständige Sprache zu bezeichnen. Zudem genieße "das Schweizerdeutsche" ein Sprachprestige und besitze eine funktionale stilistische Differenzierung wie andere Nationalsprachen. Auch dies sind für Baur Gründe, Schweizerdeutsch als eine voll ausgebaute eigenständige Sprache zu betrachten. Auch Iwar Werlen (1998) kommt nach einer differenzierten Untersuchung des Gebrauchs von Standardsprache und Schweizerdeutsch zu dem Ergebnis, dass das Konzept der Diglossie für die Sprachensituation in der Deutschschweiz nicht (mehr) angemessen sei. Obwohl sich für beide Varietäten Unterschiede hinsichtlich Produktion und Rezeption, Literalität und Oralität, massenmedialer und persönlicher Gebrauchssituation, sowie ihrer Verwendung in In- und OutgroupKommunikation konstatieren ließen, seien beide Varietäten doch voll ausgebaut. Daher müsse die Sprachensituation als asymmetrische Zweisprachigkeit charakterisiert werden. Raphael Berthele (2004) gelangt nach Überprüfung der Fergusonschen Kriterien für das Vorliegen einer Diglossiesituation zu der Ansicht, dass die Sprachensituation in der Deutschschweiz mit dem Diglossie-Konzept nur unzulänglich beschrieben ist. Zudem weist er darauf hin, dass die Mehrheit der Deutschschweizer Hochdeutsch als Fremdsprache empfinde. Aus dieser Sprachbewusstseinslage und dem soziolinguistischen Befund schließt er, dass es aus Sicht der angewandten Linguistik sinnvoller und produktiver wäre, die Deutschschweiz als bilingual zu konzeptualisieren.

Gegen die Kategorisierung des Standarddeutschen als Fremdsprache wenden sich Peter Sieber und Horst Sitta (1986: 33f). Sie betonen zwar, dass es letztlich keine linguistische, sondern eine politische Frage sei, ob Standarddeutsch in der Deutschschweiz als Fremdsprache angesehen werde; sie selbst plädieren aber trotz der besonderen Stellung der Dialekte dafür, Standarddeutsch nicht als Fremdsprache zu bezeichnen. Vor allem die feste Verankerung des Standarddeutschen in medial schriftlichen Domänen führt sie zu dieser Überzeugung. Weil 
das Ideologem, nach dem Standarddeutsch eine Fremdsprache sei, sich negativ auf die Bereitschaft zu dessen Erwerb und Gebrauch auswirke, sei es aus Sicht der angewandten Linguistik sogar geboten, dieser Haltung entschieden entgegen zu treten. Ulrich Ammon (1995: 296ff.) beschäftigt sich ausführlich mit der Frage, ob "Schwyzertütsch" eine eigenständige Sprache sei. Für ihn ist - anders etwa als für Baur - die Ausgebautheit der Dialekte kein hinreichendes Kriterium. Was ihn letztlich zu dem Ergebnis kommen lässt, dass die alemannischen Dialekte in der Deutschschweiz nicht als eigenständige Sprachen betrachtet werden können, ist ihre mangelnde Standardisiertheit, ihr zu geringer sprachsystematischer Abstand zu den (anderen) Varietäten des Deutschen, sowie die Tatsache, dass die alemannische Dialektregion nicht auf die Schweiz begrenzt ist und die schweizerdeutschen Dialekte mit den alemannischen Dialekten in Deutschland konvergieren. Haas (2004) hält trotz der sprachlichen Entwicklung vor allem in den elektronischen Medien daran fest, dass Standardsprache und Mundart in der Deutschschweiz unterschiedliche Funktionen im Sinne des Diglossie-Modells hätten. Es handle sich um einen Extremfall der Registervariation: Mundart und Standard füllten zwei prototypische Grundregister für zwei stilistische Grundfunktionen, nämlich Nähe und Distanz. Die sprachsystematische Ähnlichkeit begründe eine Sprachensituation, die der bei Zweisprachigkeit mit unähnlichen Sprachen nicht vergleichbar sei.

Es ist auffällig, dass der sprachreflexive Diskurs in der Sprachwissenschaft von denselben Dichotomisierungen beherrscht wird wie der in der Gesellschaft: Ist Standarddeutsch für Deutschschweizer Fremdsprache oder Muttersprache? Ist "Schweizerdeutsch" eine eigenständige Sprache oder ein Dialekt der historischen Gesamtsprache Deutsch? Die Debatten lassen sich immer wieder auf diese Fragen zurückführen und beziehen aus ihnen ihren Zündstoff. ${ }^{5}$ Es scheint, dass mit der Frage nach dem Fremdsprachencharakter des Standarddeutschen bzw. der Frage nach der Eigenständigkeit "des Schweizerdeutschen" die eigentliche Mitte bezeichnet ist, um die die Debatten um die Sprachensituation in der Deutschschweiz kreisen. Im Folgenden wollen wir untersuchen, warum am Ende differenzierter soziolinguistischer Analysen und ausgewogener Argumente doch immer eine Entscheidung für eine der alternativlos erscheinenden Extreme steht. Um es vorweg zu nehmen: Wir halten die Frage, ob Standarddeutsch für Deutschschweizer eine Fremdsprache ist, und die mit ihr transportierten Dichotomisierungen für nicht produktiv, wenn man sich um eine angemessene Beschreibung der Sprachensituation in der Schweiz bemüht, ja sogar für hinderlich, wenn man Konzepte für steuernde Eingriffe in die Sprachensituation im Sinne der angewandten Linguistik entwickeln möchte. Ziel dieses Aufsatzes ist es daher, die Grenzen, in denen die Debatte über die Sprachensituation in der Deutschschweiz geführt wird, aufzuzeigen und - die Komplexität der Deutschschweizer Sprachsituation berücksichtigend Vorschläge zu machen, wie diese Grenzen geweitet werden können.

Betrachtet man die unterschiedlichen Antworten auf die Frage nach dem Fremdsprachencharakter des Standarddeutschen für Deutschschweizer Sprecher, so wird

\footnotetext{
${ }^{5}$ Cf. die ideologiekritische Lesart der Diglossie-Position von Berthele (2004, vor allem 130ff).
} 
deutlich, dass zur Begründung Argumente aus drei unterschiedlichen Gebieten herangezogen werden:

1. Sprachbewusstsein: Welches Verhältnis haben Deutschschweizer zum Standarddeutschen?

2. Sprachsystematischer Abstand: Sind Standarddeutsch und das in der Schweiz gesprochene Alemannisch sprachsystematisch so verschieden, dass von zwei eigenständigen Sprachen gesprochen werden kann?

3. Erwerb des Standarddeutschen: Wird Standarddeutsch wie eine zweite Muttersprache erworben oder entspricht sein Erwerb nicht vielmehr dem einer - wenn auch früh erlernten - Fremdsprache?

Im Folgenden wollen wir jedes dieser Gebiete näher betrachten und jeweils daraufhin untersuchen, welche Gründe dafür angeführt werden können, dass Standarddeutsch für Deutschschweizer eine Fremdsprache ist. Zudem werden wir nach Ursachen suchen, warum auch die linguistische Diskussion der Sprachensituation in der Deutschschweiz immer wieder auf die Kategorie der Fremdsprache zurückgreift.

Weil die Problemstellung sehr unterschiedliche Bereiche der Sprachwissenschaft berührt, werden wir uns häufiger auf die Forschungsergebnisse anderer Wissenschaftler beziehen. Im folgenden Kapitel zum Sprachbewusstsein stützen wir uns zudem auch auf eine eigene empirische Studie, die zunächst vorgestellt werden soll.

\section{Einstellungen zum Standarddeutschen: Kollektive Ideologie und individuelles Sprachbewusstsein}

Bei dieser Studie handelt es sich um eine Fragebogenerhebung aus dem Jahr 2003, bei der 98 Personen aus der Innerschweiz, Zürich und seiner Agglomeration ${ }^{6}$ nach ihrem Gebrauch der Standardsprache und ihren Einstellungen zum Hochdeutschen befragt wurden. Im Sample waren Männer und Frauen annähernd gleich verteilt. Die Altersgruppen der unter 30-Jährigen und der Personen im Alter von 45 bis 59 Jahren sind mit einem Anteil von 40\% bzw. 30\% überproportional vertreten (cf. Tabelle 1).

\begin{tabular}{|l|c|c|}
\hline & Prozent & absolut \\
\hline unter 30 & $40 \%$ & 39 \\
\hline $30-44$ & $14,4 \%$ & 14 \\
\hline $45-59$ & $30,9 \%$ & 30 \\
\hline über 59 & $14,4 \%$ & 14 \\
\hline \multicolumn{3}{|c|}{$\mathrm{n}=98$, missing: 1} \\
\hline
\end{tabular}

Tabelle 1: Alter der Befragten

\footnotetext{
6 Mit E. Werlen (1993: 105) ist allerdings anzunehmen, dass die Problematik nicht in der gesamten Deutschschweiz gleich ist, sondern z. B. "im Kanton Bern eine stärkere Dialektfixierung besteht als im Kanton Zürich".
} 
Von den Befragten genossen rund 77\% eine Volksschulbildung und erwarben im Anschluss einen Lehrabschluss. 23\% schlossen ihre schulische Laufbahn mit der Matura ab; von ihnen setzten 91\% ihre Ausbildung mit einem Studium fort. Zwar gibt diese Stichprobe kein repräsentatives Bild der Bevölkerung in der Deutschschweiz, die Zahlen sind aber dennoch geeignet, einige Topoi des sprachwissenschaftlichen Diskurses kritisch zu beleuchten und ein differenzierteres Bild der Sprachensituation in der Deutschschweiz zu gewinnen.

In Darstellungen der Einstellungen von Deutschschweizern zum Standarddeutschen findet sich immer wieder ein gewichtiger Topos: Häufig ohne hinreichenden empirischen Befund wird behauptet, die Mehrheit der Deutschschweizer empfinde Standarddeutsch als eine Fremdsprache (Koller 1992: 41-51; Sieber/Sitta 1987: 33f.; Sieber 2001: 498ff. und zuletzt Berthele 2004: 127). Auf den ersten Blick bestätigen unsere Daten auch diesen Befund: Rund $79 \%$ der Befragten stimmten der Behauptung zu, Standarddeutsch sei für Deutschschweizer die erste Fremdsprache. Es scheint also, als hätten diejenigen, die für den Fremdsprachencharakter des Standarddeutschen plädieren, die Mehrheit der Sprecher auf ihrer Seite. Die daraus abgeleitete Forderung nach einem Kurswechsel der (angewandten) Linguistik, die endlich die Haltungen der Sprecher berücksichtigen müsse, scheint also legitim. ${ }^{7}$ Doch erlauben unsere Daten eine etwas differenziertere Analyse des Sprachbewusstseins der Befragten. Ein anderes Antwortverhalten zeigt sich nämlich, wenn man die Reaktionen auf die nun individualisierte Aussage "Hochdeutsch ist für mich eine Fremdsprache" betrachtet. Dass Standarddeutsch für sie persönlich eine Fremdsprache sei, gaben nur 30\% der Befragten an. Es gibt also offensichtlich eine tiefe Kluft zwischen der Einschätzung der allgemeinen Lage und dem persönlichen Erleben, und zwar für etwas mehr als die Hälfte der Befragten. Nur rund 28\% waren der Ansicht, dass Standarddeutsch für Schweizer im allgemeinen und auch für sie persönlich eine Fremdsprache sei, für $19 \%$ hatte Standarddeutsch überhaupt keinen Fremdsprachencharakter. Die Verteilung der Antworten auf beide Fragen zeigt Tabelle 2 .

\begin{tabular}{|c|c|c|c|c|}
\hline & & \multicolumn{2}{|c|}{$\begin{array}{l}\text { "Hochdeutsch ist für mich eine } \\
\text { Fremdsprache" }\end{array}$} & \multirow[b]{2}{*}{ Gesamt } \\
\hline & & Ablehnung & Zustimmung & \\
\hline \multirow{2}{*}{$\begin{array}{l}\text { "Hochdeutsch ist für } \\
\text { Schweizer die erste } \\
\text { Fremdsprache" }\end{array}$} & Ablehnung & $\begin{array}{c}90 \% \\
18 \\
\end{array}$ & $\begin{array}{c}10 \% \\
2 \\
\end{array}$ & $\begin{array}{c}20,9 \% \\
20\end{array}$ \\
\hline & Zustimmung & $\begin{array}{c}64,5 \% \\
49\end{array}$ & $\begin{array}{c}35,5 \% \\
27\end{array}$ & $\begin{array}{c}79,1 \% \\
76\end{array}$ \\
\hline \multicolumn{2}{|r|}{ Gesamt } & $\begin{array}{c}69,8 \% \\
67\end{array}$ & $\begin{array}{c}30,2 \% \\
29\end{array}$ & $\begin{array}{c}100 \% \\
96\end{array}$ \\
\hline \multicolumn{5}{|c|}{ Zeilenprozente, $\mathrm{n}=98$, missing: 2} \\
\hline
\end{tabular}

Tabelle 2: Fremdsprachencharakter der Standardsprache ${ }^{8}$

\footnotetext{
${ }^{7}$ Sie findet sich mit einer interessanten ideologiekritischen Lesart und schlüssig begründet in Berthele (2004: 132).

8 Lesehilfe: 64,5\% der Befragten, die der Aussage "Hochdeutsch ist für Schweizer die erste Fremdsprache" zustimmten, gaben an, für sie persönlich sei Standarddeutsch keine Fremdsprache.
} 
Ein ähnliches Antwortverhalten lässt sich auch bei der Frage nach der standardsprachlichen Kompetenz beobachten. Nur 6\% der Befragten gaben an, in der Schweiz spreche man gut Hochdeutsch. Auf die Frage "Wie gut meinen Sie, kann der durchschnittliche Schweizer Hochdeutsch?" antworteten 76\% mit "mäßig", 18\% waren gar der Ansicht, die Kompetenz ihrer Mitbürger sei "schlecht". Diese überaus negative Einschätzung muss aber bei genauerer Analyse relativiert werden. Gleicht man die Einschätzung der Kompetenz in der Deutschschweiz nämlich mit der Bewertung der eigenen Kompetenz ab, kommt man zu einem interessanten Ergebnis: Zwei Drittel der Befragten (66,3\%) schätzten ihre eigene Kompetenz besser ein als die des durchschnittlichen Deutschschweizers, 62\% hielten ihre eigene Kompetenz für gut. Individuelles Empfinden und die Einschätzung der allgemeinen Lage klaffen also auch hier weit auseinander.

Die Ergebnisse legen es nahe, eine diskursive Überformung der Wahrnehmung der Sprachensituation anzunehmen. Die Kluft zwischen persönlicher Haltung und der Annahme über die Sprachgemeinschaft ist ein starker Hinweis darauf, dass es sich bei der Einschätzung, das Standdarddeutsche sei für Schweizer einer Fremdsprache und werde entsprechend schlecht beherrscht, um ein sozial weithin akzeptiertes Stereotyp handelt, das aber auf der individuellen Ebene die Selbstwahrnehmung nur periphär beeinflusst. Es ist daher nicht mehr als ein Topos des sprachreflexiven Diskurses, wenn behauptet wird, Hochdeutsch sei für die Mehrheit der Schweizer eine Fremdsprache.

Dass ein unproblematischer Umgang mit der Standardsprache in der Deutschschweiz, insbesondere mit seinem mündlichen Gebrauch, dennoch keine Selbstverständlichkeit ist, lässt sich daran ablesen, dass beinahe die Hälfte (49\%) der Befragten angab, nicht gerne Hochdeutsch zu sprechen. Frühere Einstellungsuntersuchungen (Schläpfer/Gutzwiller/Schmid 1991; Koller 1992; Sieber 1990, 1992) konstatierten dann auch stark negative Haltungen gegenüber der Standardsprache. ${ }^{9}$ In unserer Befragung gaben rund 36\% der Befragten an, sich gehemmt zu fühlen, wenn sie Standarddeutsch sprechen; $58 \%$ erklärten, sich sicherer im Gespräch mit Nichtmuttersprachlern zu fühlen als im Gespräch mit Deutschen. Dies alles sind Indikatoren dafür, dass Standarddeutsch aber auch nicht mit der Selbstverständlichkeit einer Muttersprache gesprochen wird.

Von diesen Einstellungen betroffen ist auch das Schweizerhochdeutsche. Scharloth (2003) konnte zeigen, dass die überwiegende Mehrheit der Deutschschweizer über wenig Bewusstsein von der Plurizentrizität des Standarddeutschen verfügt. ${ }^{10}$ Für Peter von Polenz ist dies ein Grund, der Schweiz den Status eines nationalen Zentrums der deutschen Standardsprache nur eingeschränkt zuzuweisen:

Die Schweiz ist ein mehrsprachiger Nationalstaat besonderer Art; deutsche Standardsprache ist, mit drei anderen Sprachen gleichberechtigt, territorial geregelt im Gebrauch, kann aber nur in

\footnotetext{
${ }^{9}$ Eine Ausnahme bildet die Untersuchung von Hove (2002: 156-170). Die relativ positiven Einstellungen zum Standarddeutschen, die sie in ihrer Befragung erhielt, sind offenbar der Auswahl der Gewährspersonen zuzuschreiben (cf. Hove 2002: 160).

10 Auch Lorenz Hofer (1999: 14) konstatiert: "Die Existenz von Helvetismen und ihre Rolle für eine (deutsch-)schweizerische Identität im Sprachleben ist vielen Deutschschweizern und Deutschschweizerinnen kaum oder nur wenig bewusst, im Gegensatz zur Rolle der Dialekte."
} 
eingeschränkter Weise als "nationale" Varietät des Deutschen bezeichnet werden, da kaum die Standardsprache (Schriftdeutsch, Schweizerhochdeutsch), sondern eher die schweizerdeutschen Dialekte (Schwyzertütsch) als Muttersprache und nationale Identifikationssprache empfunden werden [...]. (Polenz 1999: 414)

Im Vergleich zu Österreich trifft für Deutschschweizer zwar ebenfalls zu, dass sie sich einer anderen Ausprägung des Standarddeutschen - vor allem in ihrer mündlichen Form - bewusst sind (nur 13,3\% der Befragten halten ihr Hochdeutsch für akzentfrei), weniger aber dessen, dass es sich dabei um eine eigene nationale Varietät (im Sinne Ammon 1995) handelt. Vielmehr hält man es in der Regel für eigene Unzulänglichkeit, orientiert man sich doch stark an Deutschland bzw. dessen Standardsprache. Nichtsdestotrotz werden Abweichungen vom Schweizerhochdeutschen hin zum deutschländischen Deutsch - je nach Kontext - mitunter sehr bewusst wahrgenommen. So kann deutschländisches Deutsch aus dem Mund eines Deutschschweizers durchaus als anbiedernd oder arrogant oder mindestens als unpassend empfunden werden (cf. mit Beispielen dazu Ammon 1995: 303f.). Weniger markant sind Teutonismen wohl schriftlich, dennoch werden auch sie bisweilen als störend wahrgenommen und ersetzt, genauso wie Helvetismen in einem Kontext, der nichts mit der Schweiz zu tun hat, möglichst vermieden werden (cf. dazu Beispiele in Hägi 2000).

Trotz des Bewusstseins einer sprachlichen Differenz hat sich im kollektiven Sprachbewusstsein der Deutschschweizer die Einsicht also noch nicht verbreitet, dass Schweizerhochdeutsch als eine nationale Varietät des Standarddeutschen in der Linguistik anerkannt ist. Entsprechend ist die Mundart das Symbol der regionalen Identität der deutschsprachigen Schweiz: 76\% der Befragten waren der Meinung, dass Schweizerdeutsch und nicht Standarddeutsch die Sprache der Deutschschweizer sei. Rund zwei Drittel (66,3\%) der Befragten meinten sogar, Schweizerdeutsch solle immer gesprochen werden. Dennoch wollen die Deutschschweizer auf die Standardsprache nicht verzichten. Mehrheitlich (52\%) lehnen sie den Vorschlag ab, dass Schweizerdeutsch als Nationalsprache anerkannt werden soll. Dies hat wohl weniger mit der Befürchtung zu tun, ohne Standarddeutsch international den Anschluss zu verlieren, was nur 40,8\% der Befragten zu Bedenken gaben. Vielmehr scheint es, als sei man mehrheitlich zufrieden mit der Sprachsituation, d.h. vor allem mit der funktionalen Aufteilung der Varietäten: Nur 31,6\% wünschen mehr Standarddeutsch in den Medien und 33,7\% sind lediglich der Meinung, Deutschschweizer sollten mehr im Dialekt schreiben. Dennoch ist auffällig, dass mit Standarddeutsch bessere Chancen im Beruf assoziiert werden (zu 67,3\%) und die Mehrheit der Befragten angibt, Standarddeutsch im Beruf regelmäßig zu gebrauchen (19\% immer, 57\% manchmal, 17\% selten und nur 5\% nie).

Am Ende dieses kurzen Einblicks in die Einstellungen zum Hochdeutschen steht also die Einsicht, dass 'die Standardsprache' für Deutschschweizer weder eindeutig eine Fremdsprache ist, noch - besonders mündlich - so ungehemmt wie eine Muttersprache gebraucht wird. Trotz aller negativen Einstellungen zum Standarddeutschen wollen Deutschschweizer doch auf diese Varietät nicht verzichten und sie generell durch die Mundart ersetzen. 


\section{$3 \quad$ Standarddeutsch und die alemannischen Dialekte in der Schweiz}

Schon die Untersuchungen zum Sprachbewusstsein haben gezeigt, dass die Frage, ob Standarddeutsch für Deutschschweizer eine Fremdsprache darstellt, nur aus dem Verhältnis der Varietäten zueinander zu klären ist. Neben der offensichtlichen Dichotomie von Standard/Hoch- oder Schriftdeutsch ${ }^{11}$ auf der einen und Schweizerdeutsch/Schwyzertütsch/ Mundart/Dialekt/Alemannisch ${ }^{12}$ auf der anderen Seite ist es sinnvoll, weiter zu differenzieren. Wichtig für unsere Fragestellung ist nun weniger die Tatsache, dass Schweizerdeutsch nicht als einheitliches Idiom, sondern nur in regionalen Varietäten existiert, bei denen es allerdings durch die wirtschaftliche Vorrangstellung einiger Städte und die damit verbundene Dominanz ihrer Massenmedien Angleichungstendenzen gibt (cf. Ammon 1995: 295). Wesentlicher ist, dass auch das Standarddeutsche der Deutschschweizer keineswegs einheitlich ist, sondern als Kontinuum zwischen deutschlandnahem Standarddeutsch und sehr dialektnahem Schweizerhochdeutsch verstanden werden kann: "Die verschiedenen Lautungsstufen reichen vom 'Grossratsdeutsch' (Schriftsprache mit Dialektlautung) bis hin zu überregionaler Standardlautung" (Burri et al. 1993: 20).

Folgender Romanausschnitt verdeutlicht die bestehenden Unterschiede innerhalb des Schweizer 'Schriftdeutschs' und die mit den einzelnen Varianten verbundenen konnotative Kraft:

Sogar die Lust am Radio vergeht mir oft, vor allem wenn sich unsere Politiker auf hochdeutsch melden, ich frage dich: Wie sollen Leute, die ach- und ich-Laute nicht auseinanderhalten können, die nie etwas von Hinter- oder Vordergaumen gehört zu haben scheinen, die lebenslang unfähig sind, das $\mathrm{K}$ ein bißchen, nur ein bißchen weicher und behauchter auszusprechen, die also jede Lernbereitschaft und jede Einfühlung vermissen lassen, wie sollen solche Leute in der Lage sein, die wirklich schwierigen Probleme zu bewältigen? Es mag, ich schließe es nicht aus, den einen oder anderen geben, der weicher könnte, wenn er wollte, der aber, schlau wie er ist, wohl weiß, daß seinem Volk das Rachen-ch vertrauenswürdig scheint, wogegen es den ich-Laut als Unterwanderungsversuch empfindet. Wenn doch die Frauen wenigstens, so wie versprochen, die Politik mit weiblich weichem Urstoff anreicherten! Allein, sie gaumensegeln mit. (Werner 1998: 32)

In diesem Zusammenhang wäre auch mit E. Werlen (1993: 96) zu fragen, "ob mit einer Abnahme der Bereitschaft, hochdeutsch zu sprechen, auch eine Abnahme der Fähigkeit, hochdeutsch zu sprechen, verbunden ist".

Sicherlich sind die Nuancierungen in der Aussprache am auffälligsten, jedoch auch auf den anderen sprachlichen Ebenen zu finden. Im Bereich der Standardsprache ist deswegen für die Deutschschweiz grundsätzlich ein Schweizerhochdeutsch anzunehmen, das sich vor allem mit Ammon (1995) linguistisch als nationale Varietät des Deutschen etabliert hat. Richtig ist, dass es in der Deutschschweiz kein Kontinuum zwischen Hochdeutsch und Dialekt gibt,

11 Die Termini werden hier nicht weiter differenziert, sondern als Synonyme behandelt. Um dem Missverständnis zu entgehen, wir zielten auf soziale Hierarchie (Hochdeutsch) oder Medium (Schriftdeutsch), verwenden wir meist das wertneutralere Standarddeutsch.

12 Auch diese Termini verwenden wir als Synonyme. 
"Deutschschweizerinnen und Deutschschweizer sprechen entweder Dialekt (das tun sie meistens) oder Hochdeutsch (das tun sie eher selten) - dazwischen gibt es nichts" (Studer 2001: 5). Auch wenn eine gewisse Vermischung durchaus feststellbar ist, ${ }^{13}$ "sind die Sprachformen immer deutlich voneinander getrennt; Übergangsformen gibt es kaum und Wechsel vom Dialekt in die Hochsprache erfolgen immer sehr bewusst" (Studer 2001: 5).

Diese Feststellung stimmt dagegen nur in Bezug auf die Nutzung als Umgangssprache unter Deutschschweizern. Verschiedene Ausprägungen des Schweizerhochdeutschen sind durchaus auch in der Schweiz vorhanden, und zwar innerhalb der beiden Pole "deutschländisches Deutsch" und "dialektnahes Schriftdeutsch". Entsprechend schwierig ist es, konkrete Grenzen zu ziehen, und zwar sowohl zwischen deutschländischem Deutsch und Schweizerhochdeutsch als auch zwischen Standardsprache und Substandard (cf. entsprechende unvermeidliche Grauzonen z. B. im Variantenwörterbuch 2004). Ausschlaggebend für das Ansiedeln einer sprachlichen Äußerung eines Deutschschweizers ist zum einen der Kontext und das Zielpublikum, das man vor Augen hat, zum anderen aber auch die individuelle Kompetenz in der Standardsprache. Der Ansatz, die Standardsprache nicht als einheitliche Größe zu verstehen (auch nicht Schweizerhochdeutsch als homogene Varietät), wurde in der Diskussion zur Sprachsituation in der Deutschschweiz bisher, wenn überhaupt, nur am Rande behandelt. ${ }^{14}$ Unseres Erachtens spielt aber genau dieser Aspekt in der Frage, ob denn Standarddeutsch für Deutschschweizer eine Fremdsprache sei, eine nicht unwichtige Rolle bzw. hilft, hier weiter zu differenzieren: So hat deutschländisches Deutsch für einen Deutschschweizer eher Fremdsprachencharakter als dialektnahes Schweizerhochdeutsch.

Das Verhältnis von Standardsprache und alemannischen Dialekten wird in der Debatte um den Fremdsprachencharakter des Standarddeutschen in mehrfacher Hinsicht thematisiert. Erstens wird der Gebrauch beider Varietäten als Indikator für das Vorliegen einer Diglossieoder Bilingualismussituation genommen. Mit diesem Argument werden wir uns in Kapitel 5 beschäftigen. Zweitens wird das Verhältnis von Mundart und Standardsprache im Kontext des Erwerbs des Standarddeutschen thematisiert: Dies wird Gegenstand unserer Ausführungen in Kapitel 4 sein. Drittens werden sprachsystematischer Abstand und wechselseitige (Un-)Verständlichkeit als Argumente für oder gegen den Fremdsprachencharakter des Standarddeutschen angeführt. Was diese Argumente zur Klärung unserer Frage beitragen können, wollen wir im Folgenden erörtern.

\subsection{Sprachsystematischer Abstand}

Der Abstand zwischen Standarddeutsch und dem Alemannischen in der Deutschschweiz ist nicht leicht zu bestimmen. Erstens ist die Differenz auf unterschiedlichen sprachlichen

\footnotetext{
13 I. Werlen (1998: 29) zeigt dies an auf Schweizerdeutsch verfassten Todesanzeigen, weitere Beispiele bei Haas (2004: 92) und Berthele (2004: 121f.).

14 Darauf, dass "es (mindestens) zwei hochdeutsche Varietäten in der deutschsprachigen Schweiz [gibt]: ein deutsches Hochdeutsch und ein schweizerdeutsches Hochdeutsch", verweist bereits E. Werlen (1993: 95). Leider bezieht sie weitere Überlegungen diesbezüglich nicht in ihr anregendes Modell der Sprachwelten mit ein, da die Rolle des schweizerdeutschen Hochdeutschen bisher empirisch noch ungeklärt sei (1993: 96).
} 
Ebenen unterschiedlich groß. Zweitens ist der alemannische Dialektraum nicht einheitlich, sondern weist eine erhebliche Binnendifferenzierung auf. Schließlich ist drittens auch die Standardsprache keine einheitliche Varietät, sondern zerfällt in unterschiedliche nationale Varietäten mit unterschiedlich großem Abstand zum Alemannischen in der Schweiz. So ist Schweizerhochdeutsch phonologisch, morphologisch, lexikalisch und wohl auch syntaktisch dem Alemannischen näher als das deutschländische Standarddeutsch. Dennoch benennen Baur (1983: 21ff.), I. Werlen (1998: 25f.) und Studer (2001) einige markante Unterschiede zwischen den beiden Sprachsystemen. Es ist hier nicht der Ort, diese Unterschiede in einer Zusammenschau daraufhin zu befragen, ob sie einen hinreichend großen Abstand ergeben, der es rechtfertigen würde, Standarddeutsch als Fremdsprache zu bezeichnen. ${ }^{15}$ Für Sprachen aus unterschiedlichen Sprachfamilien wäre ein solches Verfahren sicherlich geeignet, wenn auch kaum nötig; für zwei so eng verwandte Idiome wie Alemannisch und Standarddeutsch hingegen lassen sich diese Grenzen nur schwer und stets mit dem Beigeschmack dezisionistischer, wenn nicht gar ideologischer Setzung ziehen. Die Beispiele des Niederländischen und des Lëtzebuergeschen zeigen, dass ehemals als Dialekte geltende Varietäten sich zu eigenständigen Sprachen entwickeln können. ${ }^{16}$ Dafür verantwortlich ist freilich nicht etwa ein gewachsener sprachsystematischer Abstand, sondern der Status der jeweiligen Varietäten und sprachenpolitische Entwicklungen. Das Kriterium des sprachsystematischen Abstandes kann also letztlich nicht zur Klärung der Frage beitragen, ob Standarddeutsch aus der Sicht der Deutschschweiz als Fremdsprache gelten kann.

\subsection{Wechselseitige Verständlichkeit ${ }^{17}$}

"Sie sprechen ein so schönes Schwyzertütsch, da versteh ich beinah jedes Wort." Natürlich hatte ich Hochdeutsch gesprochen. Ich war ja in Deutschland. (Moser 1998: 174)

Immer wieder bekommen Deutschschweizer nach ihrem standarddeutschen Einsatz von positiv überraschten und entsprechend begeisterten Deutschen zu hören: "Hätte nie geglaubt, dass ich so gut 'Schwyzertütsch' verstehe!" (cf. auch Studer 2001: 2). Dies ist auch in Bezug auf die Verständlichkeit des Schweizerdeutschen aus (standard)deutscher Perspektive charakteristisch. Es zeigt nämlich auf, dass in der Regel erst zunächst keine Verständlichkeit zwischen deutschländischem Hochdeutsch und Schweizer Dialekt vorausgesetzt wird, und zwar von beiden Seiten (cf. auch I. Werlen 1998: 25). Diese Erwartungshaltung wird sicherlich durch das konsequente Code-Switching, bzw. das fehlende Dialekt-StandardKontinuum hervorgerufen - bekanntlich wird Schweizerdeutsch unabhängig von sozialer

\footnotetext{
15 Auch Kloss (1976) geht in seiner Bestimmung der Begriffe Ausbausprache und Abstandsprache davon aus, dass ein geringer sprachlicher Abstand kein Hinderungsgrund sein muss, durch Ausbau eine eigene Sprache zu begründen.

$16 \mathrm{Zu}$ den unterschiedlichen sprachlichen Ausgangslagen in Luxemburg, den Niederlanden und der Schweiz cf. Ammon (1995: 297).

17 Nach wie vor gilt: "Eine angemessene Untersuchungsanlage müsste berücksichtigten, dass (a) die schweizerdeutschen Dialekte untereinander verschieden verständlich sind, und dass (b) mit Versuchspersonen zu arbeiten wäre, die native Hochdeutschsprecher ohne Kenntnisse eines Dialektes sind. Da keine derartige Untersuchung vorliegt, muss man auf andere, 'weiche' Daten zurückgreifen." (I. Werlen 1998: 24).
} 
Hierarchie und Bildung von allen ${ }^{18}$ Deutschschweizern gesprochen und entsprechend aktiv beherrscht. "Die deutschsprachige Schweiz erscheint damit als im Hinblick auf das Dialektsprechen homogene Sozialgruppe mit großer Binnenkonformität und starker Außenabgrenzung" (Ammon 1995: 291).

Die Ähnlichkeit beider Varietäten und damit ihre wechselseitige Verständlichkeit gründet nicht zuletzt im "Grossteil des Lexikons alltäglicher Unterhaltung", das beide Varietäten konstituiert (Haas 2004: 92, bzw. Christen 1998: 212ff.). Dass beide Varietäten dennoch als deutlich separiert wahrgenommen werden, führt Haas (2004: 92) im wesentlichen auf zwei Aspekte zurück: "Die Dialektalität der 'schweizerdeutschen' Äusserungen wird durch die 'Grammatik' im engeren Sinne garantiert, besonders durch Phonologie und Morphologie". Die Wirkung der Aussprache ist nicht zu unterschätzen und kann sicherlich auf den ersten Blick die Gemeinsamkeiten übertönen. ${ }^{19}$

Verständnisschwierigkeiten für Deutschsprachige, die des Schweizerdeutschen nicht mächtig sind, zeigen sich aber auch, wenn auch in deutlich kleinerem Rahmen, im schriftlichen Bereich in Bezug auf das Schweizerhochdeutsche. Dass die Pole [ \pm verständlich] nicht unbedingt den Polen [ \pm mundartlich] entsprechen müssen, sondern einzelne Passagen standardsprachlicher Texte durchaus unverständlich sein können, belegen in der Schweiz standardsprachliche, aber in Deutschland i. d. R. unverständliche, ja teilweise irreführende Wörter wie Gotte 'Taufpatin', Küngel 'Kaninchen' oder die 'falschen Freunde' Postauto 'Omnibus', Peperoni 'Paprika' und Paprika 'Peperoni'. Hier zeigt sich auch, dass das Schweizerhochdeutsche dem Schweizerdeutschen wesentlich näher steht als die deutschländische Varietät.

Dagegen kann die Verständlichkeit des Standarddeutschen für Deutschschweizer in der Regel mündlich wie schriftlich vorausgesetzt werden, beruht sie schließlich auf den rezeptiven Fertigkeiten Hören und Lesen, die in der Schule und durch den Medienkonsum vollumfänglich ausgebildet werden (cf. das folgende Kapitel). Sogar spezifisch deutschländisches Deutsch stellt Deutschschweizer aufgrund der einer plurizentrischen Sprache eigenen asymmetrischen Verhältnisse (cf. Ammon 1995: 484ff.) kaum vor Verständlichkeitsprobleme.

Lässt man sich nun als Deutscher oder fortgeschrittener Deutschlerner ${ }^{20}$ gedanklich darauf ein, Schweizerdeutsch verstehen zu wollen und dafür auch ein wenig Zeit zu investieren, steht

\footnotetext{
18 Natürlich gibt es auch hier Ausnahmen: z. B. in die Deutschschweiz Eingebürgerte, die nicht des Schweizerdeutschen mächtig sind. "So leben in der Deutschschweiz viele Personen, die den diglossischen Regeln nicht unterliegen - allerdings müssen sie sich durch einen fremden Akzent 'ausweisen'" (Haas 2004: 83).

19 Diese Erfahrung musste auch eine Kölner Erasmus-Studentin an der Universität Zürich machen, als sie im Sprachkurs "Norwegisch für Anfänger" ganz irritiert war, weil sie meinte, als Einzige noch überhaupt kein Norwegisch zu verstehen, bis die Dozentin nachfragte, ob denn überhaupt alle Schweizerdeutsch verstünden (persönliche Mitteilung von Elisabeth Mennigmann, Sommersemester 1998).

20 Vor Mittelstufenniveau wird von DaF-Lehrenden in der Regel davon abgeraten, aktiv Schweizerdeutsch zu lernen. Auch Feuz (2001) berichtet in ihrem Erfahrungsbericht von erwarteten guten 
einem nicht mehr viel im Weg. Feuz (2001) hat in ihrem Erfahrungsbericht dargestellt, mit wie wenig Aufwand Dialektkurse überraschend viel erreichen: "Abbau von Hemmungen und Verminderung des Fremdheitsgefühls, Erleichterung von Kontakten und schliesslich die angestrebte Verbesserung der Integration". Diesen hohen Lerneffekt erklärt Feuz (2001) zum Einen durch die Motivation, die tatsächliche Anwendbarkeit im Alltag und dadurch, dass es sich in der Regel um lerngewohnte Lerner handelt: "Die meisten haben zuvor mindestens eine weitere Fremdsprache gelernt (oft ist es standarddeutsch)"21. Zum anderen aber "genügen schon wenige, einfache 'Transformationsregeln', die gleich zu Beginn des Kurses gelernt werden, um die Nähe des Dialekts zur Standardsprache aufzuzeigen", während "am Anfang die dialektalische Varietät sehr wohl als Fremd-Sprache empfunden wird, die gar nicht oder nur wenig verstanden wird" (Feuz 2001).

In Bezug auf das wechselseitige Verständnis zeigt sich also einerseits eine klare Asymmetrie: Bei Deutschschweizern kann (auch deutschländisches) Standarddeutsch-Verstehen vorrausgesetzt werden, umgekehrt gilt das für Deutsche bzw. Deutschlernende nicht unbedingt, besonders hinsichtlich des Alemannischen, viel weniger ausgeprägt aber auch in Bezug auf das Schweizerhochdeutsche. Andererseits wird deutlich, wie stark es sich bei der Frage, ob Standarddeutsch eine Fremdsprache sei, um eine Frage nach der entsprechenden Einstellung handelt. Grundsätzlich ist fehlende Verständlichkeit sicherlich kein Kriterium, das eine Varietät automatisch zur Fremdsprache deklariert, wie das Arabische in seinen unterschiedlichen Ausprägungen oder die sprachhistorisch und typologisch verschiedenen Sprachfamilien angehörenden 'Dialekte' des Chinesischen zeigen. Auch Sprecher eines niederdeutschen und eines bairischen Dialekts könnten einander nicht verstehen, hätten sie nicht beide die Standardsprache gelernt. Genauso wenig existiert Verständlichkeit nur innerhalb der gleichen Sprache, wie beispielsweise das Portugiesische und das Rätoromanische belegen. Im Parlament der Tschechoslowakei etwa wurde zwischen Tschechisch und Slowakisch, deren sprachliche Eigenständigkeit nie bestritten wurde, nicht gedolmetscht, und auf skandinavischen Konferenzen verstehen Schweden, Dänen und Norweger einander ebenfalls ohne größere Probleme. Die wechselseitige Verständlichkeit beider Sprachen ist demnach kein Kriterium dafür ob es sich um Varietäten einer Gesamtsprache handelt oder um eigenständige Sprachen.

\section{$4 \quad$ Praxis des Standarddeutscherwerbs}

Neben ihrer jeweiligen Muttersprache müssen alle Schweizer Schulkinder noch eine zweite Landessprache erlernen. Das ist in einem Vielvölkerstaat keine unbillige Forderung, aber für die geplagten Deutschschweizer Kinder bedeutet dies eine doppelte Belastung, denn neben ihrer eigentlichen Muttersprache, dem Schwizerdütsch, müssen sie zunächst, um lesen und schreiben zu können, das Hochdeutsche wie eine Fremdsprache erlernen, bevor sie sich mit Französisch oder Italienisch befassen können. (Bilton, Die Schweizer pauschal, 105)

\footnotetext{
Standarddeutschkenntnissen. Bereits in der Grundstufe können jedoch schweizerdeutsche Hörübungen zum 'Vertrautwerden' durchaus angebracht sein (cf. Clalüna-Hopf/Plettenberg 1988; Maurer 2000).

21 Dies impliziert, dass es sich für Feuz bei Schweizerdeutsch und Standarddeutsch um zwei verschiedene Sprachen handelt!
} 
Im Folgenden wollen wir fragen, ob Zeitpunkt, Art und Strategien des Erwerbs bzw. des Erlernens und der Vermittlung des Standarddeutschen Indikatoren für dessen Fremdsprachencharakter sind. Wir wollen untersuchen, wann welche Art standardsprachlicher Kompetenz erworben wird, um zu überprüfen, ob diese Prozesse denen beim Erwerb prototypischer Fremdsprachen entsprechen. Allgemein ist davon auszugehen, dass Deutschschweizer Kinder zuerst alemannisch sprechen können, ehe sie zwischen zwei und vier Jahre später (Häcki Buhofer et al. 1994: 150) Kompetenz im Standarddeutschen erwerben. Dieser Erwerb setzt aber schon vor der Schulzeit ein und führt zu passiver wie aktiver Kompetenz. Bei diesem Erwerb spielen neben dem Vorlesen die audiovisuellen Massenmedien wohl eine wichtige Rolle, vor allem Fernsehen und Video (Böhme-Dürr 1994: 50). ${ }^{22}$ Die Art des Erwerbs interpretieren Wissenschaftler unterschiedlich: Während die einen den Aufbau standardsprachlicher Kompetenz als erweiterten Erstspracherwerb mit einigen Zügen von Zweitspracherwerb ansehen (Häcki Buhofer/Burger 1998: 137), betonen andere jene Erwerbsstrategien der Lerner, die sich auch beim Zweitspracherwerb beobachten lassen (Stern 1988: 148; Berthele 2004: 122ff.). Es handelt sich also offenbar um eine Mischform: Auf dem Gebiet von Morphologie und Syntax finden in größerem Maße Transferprozesse statt und sind häufiger Interferenzen beobachtbar, während die Lexik davon weit gehend unberührt bleibt. Zudem finden Mundart- und Standardspracherwerb nicht völlig unabhängig von einander statt: Obwohl der Standardspracherwerb später einsetzt, werden - insbesondere im Bereich der Lexik - Mundart- und Standardkompetenz gemeinsam und im Austausch entwickelt (Häcki Buhofer et al. 1994: 150).

In der Schule wurde bislang erst ab dem dritten Schuljahr im Unterricht einigermaßen konsequent Standarddeutsch gesprochen. Dies entspricht etwa dem Alter, in dem in anderen Ländern mit der schulischen Vermittlung prototypischer Fremdsprachen begonnen wird. Im Unterschied zur Situation beim Erlernen prototypischer Fremdsprachen verfügen aber die Schüler bereits über eine relativ weit reichende Kompetenz. Die Schule trägt dem Rechnung, indem im Unterricht eine Mischung aus gezielter Vermittlung standardsprachlicher Kompetenz im Sinne eines Lernens und einem auf ungesteuerten Erwerb zielenden sprachlichen Handeln praktiziert wird. Wenige grammatikalische und lexikalische Besonderheiten des Standarddeutschen werden im Deutschunterricht gezielt und teilweise kontrastiv eingeführt. Auch diese Vermittlungspraxis ist für prototypische Fremdsprachen sicherlich seltener. $^{23}$ Bemerkenswert ist, dass der Erwerb aktiver sprechsprachlicher Kompetenz durch die Schule offenbar gebremst wird. Es kommt zur Ausbildung negativer Einstellungen (Häcki Buhofer/Burger 1998: 138; Sieber/Sitta 1986: 143ff., 1994: 201; Sieber 2001: 498f.), vor allem im Übergang vom 7. zum 8. Lebensjahr (Häcki Buhofer et al. 1994: 167), die den Gebrauch auf wenige stark geregelte Domänen einschränken.

\footnotetext{
${ }^{22}$ In unserer Befragung gaben aber immerhin 20\% der Befragten an, Standarddeutsch vor der Schulzeit in der Familie, 25\% durch Kontakt mit Deutschen vermittelt bekommen zu haben. (Mehrfachnennungen waren möglich.)

${ }^{23}$ Allerdings werden solche Ansätze heutzutage durchaus auch in der Fremdsprachendidaktik propagiert.
} 
Die Verhältnisse beim Standardspracherwerb sind demnach so vielschichtig, dass es weder sinnvoll ist, von einem reinen Zweitspracherwerb, noch von einem reinen Erstspracherwerb zu sprechen. Weder Erwerbsalter noch Erwerbsart noch Vermittlungsmethode machen es plausibel, dass Standarddeutsch für Deutschschweizer den Charakter einer Fremdsprache hat. Aber auch der Umkehrschluss wäre voreilig: Weil Situationen selten sind, in denen Deutschschweizer Kinder im Alltag mit Kommunikationspartnern standardsprachlich interagieren können, ist der Erwerb einer aktiven Kompetenz kaum mit der beim prototypischen Erstspracherwerb zu vergleichen.

\section{$5 \quad$ Bilingualismus oder Diglossie? - Zur Gretchenfrage der Deutschschweizer Soziolinguistik}

Die Beschreibungen der Sprachensituation in der Deutschschweiz lassen sich in zwei Klassen einteilen: Das eine Lager konstatiert eine Diglossiesituation, das andere hält die Deutschschweiz für einen Musterfall des Bilingualismus (cf. Kapitel 1). Um zu verstehen, welche Kriterien bei der Bestimmung der Sprachensituation zur Anwendung kommen und welche Implikationen die jeweiligen Konzepte für die Frage nach dem Fremdsprachencharakter des Standarddeutschen haben, ist zunächst eine Bestimmung beider Termini von Nöten. Zwar ist "Diglossie" in etymologischer Perspektive das griechische Pendant des lateinischen "Bilingualismus" und bedeutet dem Wortsinn nach ebenfalls "Zweisprachigkeit" (Kremnitz 1987: 209); doch verbergen sich hinter den unterschiedlichen Wortformen auch unterschiedliche Konzepte mit unterschiedlicher wissenschaftsgeschichtlicher Herkunft.

Das Konzept des Bilingualismus bzw. Multilingualismus (auch Zwei- bzw. Mehrsprachigkeit) hat seinen Ursprung in der Spracherwerbsforschung und diente zunächst nur der Bezeichnung der Kompetenz mehrsprachiger Individuen. Als mehrsprachig gilt in der gegenwärtigen Forschung, "wer sich irgendwann in seinem Leben im Alltag regelmäßig zweier oder mehrerer Sprachvarietäten bedient und auch von der einen in die andere wechseln kann, wenn dies die Umstände erforderlich machen" (Lüdi 1996: 234). Bald jedoch erfuhr das Konzept eine Ausweitung auf ganze Sprachgemeinschaften, in denen mehrere Sprachen Verwendung fanden. ${ }^{24}$ Im Unterschied zu einem individuellen Multilingualismus wird dann von einem kollektiven Multilingualismus gesprochen, ein Konzept, das weitere Differenzierungen erfahren hat. Michael Clyne (1997: 301) unterscheidet einen offiziellen und einen de-factoMultilingualismus. Offiziell multilinguale Gesellschaften werden zu solchen durch Gesetz oder Anordnung erklärt, etwa dadurch, dass verschiedene Amtssprachen bzw. Nationalsprachen zugelassen werden. Dies kann auch der Fall sein, wenn eine Gemeinschaft aus territorial monolingualen Einheiten mit je unterschiedlicher Sprache besteht. Es ist also für den offiziellen kollektiven Multilingualismus nicht nötig, dass die Sprecher tatsächlich mehrere Sprachen beherrschen. Dies unterscheidet den "offiziellen" Multilingualismus vom

\footnotetext{
24 Manche Soziolinguisten verwenden für die Sprachensituation in einer Gesellschaft den Terminus Multilingualismus, für die Kompetenz eines Sprechers in mehreren Sprachen die Bezeichnung Bilingualismus (Trudgill 2003: 15).
} 
de-facto-Multilingualismus: Letzterer liegt nur dann vor, wenn eine Vielzahl der Sprecher tatsächlich mehrere Sprachen beherrschen. Diese Erweiterung des Konzeptes individueller Mehrsprachigkeit um ein Konzept der gesellschaftlichen Mehrsprachigkeit macht eine Anwendung des Bilingualismusbegriffs auf die Deutschschweiz erst möglich. Wenn die Sprachensituation in der Deutschschweiz als bilingual charakterisiert wird, dann ist damit zunächst einmal ganz allgemein gemeint, dass dort zwei Sprachen im Sinne eines de-factoBilingualismus neben einander existieren: Alemannisch und Standarddeutsch.

Anders als das Konzept des Multilingualismus wurde der moderne Diglossiebegriff in der Soziolinguistik geprägt. Als sein Vater gilt Charles Ferguson, der ihn auf Sprachensituationen angewendet wissen wollte, "where two varieties of a language exist side by side throughout the community, with each having a definite role to play" (Ferguson 1959: 325). Die spezifische Rolle, die den jeweiligen Varietäten zukommt, bestimmt Ferguson hinsichtlich der Dimensionen Funktion, Prestige, literarisches Erbe, Erwerb, Standardisiertheit, Stabilität, Komplexität des grammatischen Systems, Lexik und Phonologie. Er geht davon aus, dass Diglossie dann vorliegt, wenn "in addition to the primary dialects of the language [...], there is a very divergent, highly codified (often grammatically more complex) superposed variety, the vehicle of a large and respected body of written literature [...] which is learned largely by formal education and is used for most written and formal spoken purposes but is not used by any sector of the community for ordinary conversation" (Ferguson 1959: 336).

Weil Ferguson sein Diglossiekonzept unter anderem am Beispiel der Deutschschweiz entwickelte, galt diese lange Zeit als Musterfall einer Diglossiesituation. Doch wurde Fergusons Beschreibung der Sprachensituation in der Deutschschweiz Zielscheibe berechtigter Kritik. ${ }^{25}$ Der Wandel in der funktionalen Verteilung von Dialekt und Standardsprache ließ Kolde (1981) zu dem Schluss kommen, dass die Sprachensituation adäquater als mediale Diglossie beschrieben werden müsse. Er begründete dies damit, dass in den meisten Fällen das Medium die Wahl der Varietät bestimme. Nicht zuletzt der Sprachgebrauch in den neuen (elektronischen) Medien hat aber auch diese Bestimmung der Funktionsverteilung fragwürdig werden lassen. Ris (1990: 42) kam zu dem Ergebnis, dass die alemannische Mundart in der Deutschschweiz nahezu alle Funktionen hat, die in anderen Ländern der Standardsprache zukommen. Damit war die wichtigste Bedingung für das Vorliegen einer Diglossiesituation nicht mehr gegeben: die annähernd komplementäre funktionale Verteilung der beiden Varietäten. Für Ris war daher klar, dass Standarddeutsch als Zweitsprache im Sinne des Bilingualismus-Modells zu werten sei.

Auch wenn Ris nicht nur Zustimmung fand, wäre doch die Konstatierung einer funktionalen Äquivalenz von Standarddeutsch und alemannischer Mundart im Bereich der gesprochenen Sprache und vermehrt auch im Medium der Schriftlichkeit nicht weiter problematisch und würde die hier aufgeworfene Frage nach dem Fremdsprachencharakter des Standarddeutschen nicht berühren. Dennoch stellen die Verfechter der Bilingualismus-Hypothese immer wieder fest, Standarddeutsch und das Alemannische in der Schweiz seien als verschiedene Sprachen

${ }^{25} \mathrm{Zu}$ den jeweiligen Akzentverschiebungen in der Debatte cf. auch Haas (2004: 84f.). 
zu werten (I. Werlen 1998: 24; Berthele 2004: 127). Warum, so muss man zugespitzt fragen, wird aus der funktionalen Äquivalenz zweier Varietäten in einer de-facto-BilingualismusSituation abgeleitet, es handle sich bei der einen für die Sprecher um die Muttersprache, bei der anderen um eine Fremdsprache? Die Antwort liegt auf der Hand: Mit der terminologischen Entscheidung für das Bilingualismuskonzept ist stillschweigend auch eine Entscheidung für den Fremdsprachencharakter des Standarddeutschen gefallen. Während Ferguson (1995: 336) in seiner klassischen Bestimmung des Diglossie-Begriffs davon ausgeht, dass die beiden funktional unterschiedlichen Idiome Varietäten derselben Sprache sind (cf. auch Hoffmann 1991: 166), findet sich in klassischen Definitionen des Bilingualismus-Begriffs die Bestimmung, dass es sich bei beiden Varietäten um verschiedene Sprachen handeln muss (cf. die Definitionen von Weinreich und Bloomfield, zitiert in Hoffmann 1991: 15). Die Ablehnung einer funktionalen Verteilung beider Varietäten rechtfertigt also die Wahl, die Sprachensituation in der Schweiz als bilingual zu konzeptualisieren. Die Entscheidung für das Konzept des Bilingualismus impliziert dann, dass es sich bei den Idiomen um eigenständige Sprachen handeln muss. Da das Alemannische die zuerst erworbene Sprache ist, muss schließlich - häufig in einer Gleichsetzung von Fremdund Zweitsprache - der Fremdsprachencharakter des Standarddeutschen konstatiert werden. ${ }^{26}$

Wir glauben, dass hier die wissenschaftsgeschichtliche Tradition des BilingualismusKonzeptes zu einem Schluss verleitet, der durch den linguistischen Befund nicht gerechtfertig ist. Wie bereits erwähnt, wurde der Begriff des Bilingualismus im Kontext von linguistischen Untersuchungen geprägt, in denen es um den Erwerb der Sprache des Gastlandes durch Immigranten (Bloomfield) oder um den Kontakt von Sprachen, bei denen es sich nicht um Varietäten derselben historischen Gesamtsprache handelt (Weinreich), ging (cf. Hoffmann 1991:15). Diese Einschränkung des Bilingualismus-Begriffs auf systematisch deutlich verschiedene Sprachen muss aber als überholt gelten. Neuere Definitionen enthalten das Kriterium der Fremdsprachigkeit der beiden in einer Bilingualismussituation vorhandenen Idiome dann auch nicht mehr. Als mehrsprachig gilt demnach, "wer sich irgendwann in seinem Leben im Alltag regelmäßig zweier oder mehrerer Sprachvarietäten bedient und auch von der einen in die andere wechseln kann, wenn dies die Umstände erforderlich machen, aber unabhängig von der Symmetrie der Sprachkompetenz, von den Erwerbsmodalitäten und von der Distanz zwischen den beteiligten Sprachen." (Lüdi 1996: 234)27

\footnotetext{
26 Die Bezeichnung des Standarddeutschen als Fremdsprache und die Betonung der Eigenständigkeit der alemannischen Mundart in der Schweiz führt zu teils kuriosen terminologischen Schwierigkeiten: Man spricht von "Schweizerdeutsch", stellt aber in Abrede, dass es sich dabei um eine Varietät des Deutschen handelt. Eine solche Situation gibt es allerdings auch bei "Weißrussisch" einschließlich der Eigenbezeichnung "belaruski", das aber eindeutig keine Varietät des Russischen (ruski) ist, sowie historisch bei duits (=deutsch), das im Niederländischen vor der Ersetzung durch nederlands auch die eigene Sprache bezeichnete (was noch heute an der englischen Bezeichnung Dutch zu erkennen ist).

27 Abgesehen von der u. E. terminologisch-konzeptuell determinierten Entscheidung dafür, Standarddeutsch als Fremdsprache zu kennzeichnen, ist der Schluss auf den Fremdsprachencharakter innerhalb der Argumentation auch nicht kohärent. Die Vertreter der Bilingualismus-Hypothese werden nämlich nicht müde zu betonen, dass es keine klaren sprachlichen Kriterien gibt, die eine Entscheidung rechtfertigen, ob eine Varietät zu einer historischen Gesamtsprache gehört oder nicht, ja, dass diese Entscheidung im Falle des Alemannischen in der
} 
Ähnlich verhält es sich mit dem Diglossie-Konzept. Auch hier ist das Kriterium, nach dem die beiden Idiome Varietäten derselben Sprache sein müssen, nicht mehr gültig:

Heute spricht man auch dann geläufig von Diglossie, wenn die Varietäten nicht derselben Sprache angehören [...], wenn die Komplementarität nicht dem üblichen Schema High vs. Low folgt [...], wenn die Situation weniger stabil ist [...] und/oder keine 'nationalen' Gesellschaften, sondern nur kleine Minderheiten betrifft, und unabhängig davon, ob alle Sprecher oder nur eine kleine Minderheit von ihnen beide Varietäten beherrschen. (Lüdi 1996: 237)

Aus der Bestimmung der Deutschschweizer Sprachensituation als diglossisch ergibt sich demnach nicht zwingend, dass Standarddeutsch und Alemannisch Varietäten derselben Sprache seien. ${ }^{28}$

Nimmt man aber dem Diglossie-Begriff seine Beschränkung auf Varietäten derselben Sprache und dem Bilingualismus-Begriff das Kriterium eines großen sprachsystematischen Abstandes, stehen die beiden Konzepte sich nicht mehr als entgegengesetzte Pole einer zweidimensionalen Achse gegenüber. Schon Fishman (1975: 95ff.) hatte das komplexe Verhältnis von Bilingualismus und Diglossie systematisiert und die Möglichkeiten und Bedingungen des gemeinsamen Auftretens von Bilingualismus und Diglossie innerhalb einer Sprachgemeinschaft beschrieben. Auch wenn man neuere Definitionen beider Konzepte betrachtet, wird deutlich, wie wenig beide als unvereinbare Alternativen betrachtet werden. Etwa definiert Clyne (1997: 302) "Diglossie", indem er sie als Sonderfall des gesellschaftlichen Multilingualismus bestimmt:

In multilingual societies, in which the same languages are generally used by the same people, the various languages have different functions. This situation is known, depending on the number of languages involved, as diglossia, triglossia, or polyglossia.

Es ist also die klare funktionale Distribution unterschiedlicher Varietäten, die es erlaubt, bei Bi- oder Multilingualismus von Diglossie zu sprechen (cf. hierzu auch Weisgerber 1996: 266, Romaine 2000: 46ff). Clyne vertritt die Ansicht, dass immer, wenn gesellschaftlicher defacto- Multilingualismus vorliegt, auch Diglossie vorhanden ist. Dabei ist es gleichgültig, ob es sich um Varietäten derselben Sprache handelt oder um eigenständige Sprachen.

Gegen die Aufgabe des Kriteriums der Verwandtschaft der in einer Diglossiesituation vorkommenden Sprachen wendet sich Walter Haas (2004: 88). In seiner innovativen und richtungsweisenden Deutung der Sprachensituation in der Deutschschweiz argumentiert er, dass die sprachsystematische Ähnlichkeit beider Varietäten erst jene funktionale Distribution ermöglich, die für das Vorliegen einer Diglossiesituation charakteristisch seien. Doch auch mit diesem Argument ist die Frage nach dem Fremdsprachencharakter des Standarddeutschen

\footnotetext{
Schweiz und des Standarddeutschen nicht möglich ist. Das für Bilingualismus-Situationen konstitutive Charakteristikum, nach der die beiden Sprachen keine Verwandtschaft besitzen, bedient sich aber implizit des sprachsystematischen Abstands als Kriterium; eines Kriteriums also, das die Vertreter der BilingualismusHypothese verworfen hatten. Es ist demnach weder notwendig noch kohärent, Standarddeutsch in der Deutschschweiz zur Fremdsprache zu erklären.

28 Gegen die Aufgabe des Kriteriums wendet sich aber Haas (2004: 91) cf. unten.
} 
nicht entschieden, denn aus der sprachsystematischen Ähnlichkeit folgt nicht, dass es sich bei Mundart und Standardsprache in der Deutschschweiz um Varietäten derselben Sprache handle. Das Vorliegen einer großen sprachsystematischen Ähnlichkeit wird auch von niemandem in der Debatte um die Sprachensituation in Frage gestellt, ganz gleich, ob man für oder gegen den Fremdsprachencharakter des Standarddeutschen argumentiert.

Zusammenfassend lässt sich also sagen, dass die wissenschaftliche Debatte um die adäquate Bestimmung der Sprachensituation in der Deutschschweiz immer wieder auf eine Entscheidung zwischen den Kategorien Bilingualismus oder Diglossie hinausläuft und damit häufig eine Entscheidung für oder gegen den Fremdsprachenstatus des Standarddeutschen verknüpft ist. Daran sind zwei Dinge problematisch: Erstens sind Bilingualismus und Diglossie nicht zwei entgegengesetzte Pole einer zweidimensionalen Achse. Die Diskussion über die Beschreibung der Sprachensituation in der Deutschschweiz gibt aber vor, sie seien es, und lässt sich als eine wechselnde Positionierung auf dieser Achse beschreiben. Problematisch ist zweitens die Gleichsetzung von Bilingualismus und dem Fremdsprachencharakter der Zweitsprache. Wie wir gezeigt haben, findet sich in neueren Bestimmungen des Bilingualismus-Begriffs nicht mehr das Kriterium, wonach die beiden Idiome Varietäten unterschiedlicher Sprachen sein müssen. Beschreibt man die Sprachensituation als gesellschaftlichen Bilingualismus, bedeutet dies also nicht automatisch, dass es sich bei Standarddeutsch um eine Fremdsprache handelt. Umgekehrt folgt aber aus der Bestimmung der Sprachensituation als diglossisch auch nicht, dass Standarddeutsch und die alemannischen Dialekte in der Deutschschweiz Varietäten derselben Sprache sind.

\section{$6 \quad$ Fazit}

\subsection{Aus Sicht der angewandten Linguistik}

Die (auch) deutschsprachige Schweiz wird in einem etwas unpräzisen Sprachgebrauch zur Gruppe der deutschsprachigen Staaten gezählt. Sie nimmt aber darin eine Sonderstellung ein a) durch ihre Viersprachigkeit und b) durch den Stellenwert des Dialekts. (Langner 2001:108)

Langner weist zwar in seinem Artikel darauf hin, dass die gängige Unterscheidung der Fremdsprachendidaktik in Deutsch als Fremdsprache ("als Begriff für den sprachlichen Lerngegenstand Deutsch nicht deutschsprachiger Lernender, die institutionell gesteuert im Ausland Deutsch lernen und für die diese Sprache nicht unmittelbar kommunikativ relevant ist") und Deutsch als Zweitsprache (als Begriff für "den sprachlichen Lerngegenstand Deutsch nicht deutschsprachiger Lernender, aber in deutschsprachiger Umgebung, eher ungesteuert, und die Sprache ist für sie unmittelbar kommunikativ relevant") für die Schweiz problematisch sei.29 Dennoch werden diese Begriffe verwendet: DaF für die nichtdeutschsprachige Schweiz im Unterschied zu DaZ in der deutschsprachigen Schweiz. Der dargelegte für die deutschsprachige Schweiz fremdere Charakter der Standardsprache mit

29 "Diese Unterscheidung ist für ein mehrsprachiges Land mit dem Deutschen als einer Landessprache problematisch, da es hier häufig zu Kontaktsituationen kommt, in denen die gelernte Fremdsprache sehr wohl kommunikativ relevant ist." (Langner 2001:109). 
deutschländischer Norm zeigt nun einerseits auf, wo genau diese Unzulänglichkeiten liegen, und andererseits, welche Konsequenzen daraus zu ziehen sind. Für nichtdeutschsprachige Lerner ist Standarddeutsch in der Deutschschweiz nur bedingt Zweitsprache, weil die Umgebung nur sehr eingeschränkt standardsprachlich ist. In Bezug auf die mündliche Kommunikation ist es partiell eine Fremdsprache, was in Deutschkursen auch regelmäßig Frustration auslöst, da die gelernte Sprache nicht unbedingt angewandt werden kann (cf. dazu Feuz 2001). Dies heißt wiederum, dass Standarddeutsch für Deutschschweizer auch nicht ohne Probleme als Muttersprache klassifiziert werden kann, nämlich nicht in Bezug auf die gesprochene Umgangssprache: Hier wäre Standarddeutsch für Deutschschweizer eine Art Zweitsprache (wohl weniger Fremdsprache im engeren Sinne, da die Umgebung vor allem durch die Medienpräsenz durchaus deutschsprachig ist), denn: "Zwischen quasi muttersprachlichem Sprachvermögen und Fremdsprachenkenntnissen ist die Verfügung über eine Sprache als Zweitsprache angesiedelt" (Oomen-Welke 2003:146). Für den muttersprachlichen Deutschunterricht heißt das also, dass die standardsprachliche mündliche Ausdrucksfähigkeit gezielter und bewusster vermittelt werden müsste, und zwar anders als die anderen Fertigkeiten. Bewusster werden sollte man sich auch in Hinblick auf die standardsprachlichen Varietäten: Unseres Erachtens spielt es wahrscheinlich weniger eine Rolle, ob man sich explizit für die deutschländische Varietät oder das Schweizerhochdeutsche entscheidet, als dass man sich überhaupt festlegt und einen entsprechenden Rahmen schafft. Erstere hätte aufgrund der größeren Distanz den Vorteil, dass man die Verschiedenheit, analog zum Englischen beispielsweise, bewusster wahrnimmt und vielleicht leichter auf entsprechende bewährte Methoden des gesteuerten Fremdsprachenerwerbs zurückgreifen könnte. Hingegen hätte das Schweizerhochdeutsche den Vorteil, dass es einem Deutschschweizer in der Regel näher und vertrauter ist und wohl zu einem großen Teil schon umgesetzt wird. Zudem würde das Wissen um die Plurizentrizität des Deutschen im Sprachbewusstsein verankert werden. ${ }^{30}$ Nicht jedes von Deutschschweizern gesprochene Hochdeutsch ist aber tatsächlich normgerecht, also der schweizerhochdeutschen Sprachkonvention entsprechend (cf. dazu Hove 2002: 6ff.) - hier müssten klarere Vorgaben gemacht werden, damit wirkliche sprachliche Mängel behoben werden können: Einfach nur in allen Fächern "Standarddeutsch" als Unterrichtssprache anzusetzen, greift jedenfalls zu kurz und überfordert bisherige Kompetenzen (cf. E. Werlen 1993: 102f.). Nicht zuletzt muss ein Bewusstsein für Schweizerhochdeutsch und seine (Gleich-)Berechtigung neben dem deutschländischen Standard geschaffen werden (Bickel 2001) - und zwar nicht nur als AlibiGröße - und damit ein selbstbewussterer und selbstverständlicherer Umgang mit der Standardsprache auch im mündlichen Ausdruck. Wie Berthele (2004: 112) aufzeigt, gibt es "keinen solchen intrinsischen Zusammenhang zwischen Abneigung gegenüber der Hochsprache und ihrem Fremdsprachenstatus" (cf. dagegen Sieber/Sitta 1986: 34). Vielmehr

\footnotetext{
${ }^{30}$ So unterschied und lernte der in Wiedenbrück (Nordrhein-Westfalen) geborene und in Glarus (Schweiz) aufgewachsene Schriftsteller Tim Krohn als Kind nicht nur "eine hochdeutsch genannte Kriegswirrenmischung aus Hamburgisch, Ostpreussisch und Bühnenhochdeutsch, wie es meine Eltern sprachen, und Glarnerdeutsch, wie es alle anderen gebrauchten", sondern sprach in der Schule auch "dieselbe helvetisch gefärbte 'Schriftsprache' wie alle Kinder" (Krohn 1998: 168).
} 
ist es wohl die Diskrepanz zwischen der Wahrnehmung und den Einstellungen der Deutschschweizer, die überfordert, da sie deutschländisches Hochdeutsch aufgrund seiner Extranormativität und seiner vorwiegend medialen Vermittlung als fremder empfinden, Schweizerhochdeutsch aber aufgrund seiner mundartlich beeinflussten Eigenheiten, seiner Symbolisierung schweizerischer Identität und seiner Vermittlung auch in Face-to-FaceInteraktionen als vertrauter empfinden. Das Problem liegt unseres Erachtens darin, dass im Unterricht die Anforderungen nicht explizit an einer der beiden Varietäten orientiert werden. Ein geklärteres, sachlicheres Verhältnis diesbezüglich, entsprechend verbesserte Kompetenzen und geschaffenes Prestige könnten auch die Kommunikation mit nichtdeutschsprachigen Schweizern wesentlich entkrampfen. Wenn man mit gleichen offenen Karten und expliziter Erörterung der Standardvarietäten in der Westschweiz den Deutsch-alsFremdsprache-Unterricht gestaltete, könnte auch dieser profitieren.

\begin{abstract}
Nun sind Einstellungen als relativ feste, nicht ohne weiteres und nur in Grenzen veränderbare mentale Konstrukte, die das Handeln - und das heißt hier auch: das unterrichtliche Handeln prädisponieren, ernst zu nehmen, und zwar auch dann, wenn sie mit wissenschaftlichen Einsichten nicht im Einklang stehen. (Studer 2002)
\end{abstract}

Die Frage, welchen Stellenwert der Dialekt im Unterricht bekommen soll, ist so pauschal sicher nicht zu beantworten. Nicht zu unterschätzen ist aber sein Wert in Bezug auf den "Aufbau einer Wahrnehmungstoleranz gegenüber Varietäten des Deutschen" (Studer 2002: 5). Gerade hier hätte die Schweiz durch ihre spezifische Sprachsituation hervorragende Möglichkeiten, u. a. das bisherige Desiderat "einschlägiger Konzepte zur Integration der Dialekte in das Lehren und Lernen von Fremdsprachen allgemein und in den DaF-Unterricht besonders" (Studer 2002) beispielhaft anzugehen. Voraussetzung ist aber mitunter, dass man von festgefahrenen Konzepten Abstand nimmt und wirklich neue Wege einschlägt.

\title{
6.2 Aus Sicht der Soziolinguistik
}

Unsere Untersuchungen zur Frage, ob Standarddeutsch für Deutschschweizer eine Fremdsprache ist, haben auf unterschiedlichen Gebieten zum gleichen Ergebnis geführt. Im Sprachbewusstsein der Mehrheit der Sprecher ist Standarddeutsch keine Fremdsprache. Der sprachsystematische Abstand und die wechselseitige Verständlichkeit sind keine Kriterien für die Kategorisierung so ähnlicher Sprachen als Fremdsprachen. Alter und Praxis des Erwerbs, sowie die Strategien der Vermittlung des Standarddeutschen entsprechen nicht denen prototypischer Fremdsprachen, aber auch nicht denen prototypischer Standardvarietäten. Andererseits zeigen unsere Untersuchungen aber auch, dass Sprachbewusstsein, Erwerb und Gebrauch durchaus abweichen von anderen Sprachsituationen, in denen ein Dialekt-StandardBilingualismus vorliegt. Auch die Bewertung des sprachsystematischen Abstands und die wechselseitige Verständlichkeit rechtfertigen es nicht ohne weiteres, beide Idiome als Varietäten derselben Sprache zu klassifizieren. Wie beantworten wir also die Frage, ob Standarddeutsch für Deutschschweizer eine Fremdsprache ist oder nicht? Wir plädieren dafür, die Frage nicht zu beantworten, sondern sie zu verwerfen. Sie ist falsch gestellt, denn sie stellt den Befragten vor Alternativen, die vor dem Hintergrund der Sprachsituation in der Deutschschweiz nicht angemessen sind. Die gesellschaftliche und linguistische Debatte wird 
aber immer wieder auf diese Alternativen zurückgeworfen, obwohl differenzierte Analysen zum Status des Standarddeutschen vorliegen. Wir wollen nun vorschlagen, dieses kategoriale Prokrustesbett zu verlassen.

Wir sind der Ansicht, dass die Forschung dem besonderen soziolinguistischen Status des Standarddeutschen in der Deutschschweiz durch die Prägung einer eigenständigen Kategorie Rechnung tragen sollte, die sich zu der Frage, ob es sich um eine Fremdsprache handelt oder nicht, neutral verhält. Wir schlagen dafür das Konzept der Sekundärsprache vor. Das soziolinguistische Konzept "Sekundärsprache" ist primär bezogen auf den Status einer Sprache oder Varietät in einer Sprachgemeinschaft bzw. den Umgang der Sprecher mit diesem Idiom. ${ }^{31}$ Das Konzept ist gedanklich nicht auf den 'Sonderfall Schweiz' beschränkt, sondern lässt sich in vielleicht noch zu verfeinernder Form vermutlich auch auf viele andere Sprachsituationen anwenden, bei denen die Konzepte Diglossie und Bilingualismus in ähnlicher Weise zu kurz greifen, etwa auf den Status des Spanischen in Katalonien, des Hocharabischen in den arabischen Ländern oder auch einer Zweitsprache, in der große Teile der Bevölkerung quasi-muttersprachliche Kompetenz erworben haben, wie Russisch in Kasachstan. Die Bezeichnung Sekundärsprache ist insofern angemessen, als es die Abhängigkeit des Konzeptes vom Konzept der Primärsprache andeutet. Bei der Primärsprache handelt es sich um das von der überwiegenden Mehrheit der Sprecher zuerst erlernte Idiom. Im Unterschied zur Primärsprache erfolgt der Erwerb produktiver Kompetenz der Sekundärsprache zumindest teilgesteuert, jedoch i. d. R. nicht vollständig gesteuert wie bei prototypischen Fremdsprachen. Im Vergleich zur Primärsprache wird die Sekundärsprache von den autochthonen Sprechern untereinander seltener verwendet, gehört jedoch im Gegensatz zu einer Fremdsprache durchaus zum Alltag. Die produktive Kompetenz in der Sekundärsprache unterscheidet sich von der in der Primärsprache hinsichtlich der Anzahl der stilistischen Register, über die die Sprecher verfügen. Dies wirkt sich auch auf die Einschätzung der eigenen Fähigkeiten aus: Während die Selbsteinschätzung der rezeptiven Kompetenz bei Primär- und Sekundärsprache gleich ist, halten die Angehörigen der Sprachgemeinschaft häufig ihre eigene produktive Kompetenz für defizitär. Die Sekundärsprache ist immer voll ausgebaut und standardisiert und hat eine größere kommunikative Reichweite als die Primärsprache. Die Verteilung des Sprachprestiges ist stark abhängig von der Gebrauchssituation. Affektiv steht die Primärsprache für Vertrautheit, Nähe, Wärme und gilt als emotionale Heimat, der Gebrauch der Sekundärsprache ist dagegen verbunden mit Funktionalität, Formalität oder Distanz. Im Unterschied zur Sekundärsprache symbolisiert die Primärsprache lokale, regionale oder gar nationale Identität.

31 Dies unterscheidet es von dem in der Spracherwerbsforschung selten verwendeten Konzept der Sekundärsprache. 


\begin{tabular}{|c|c|c|c|}
\hline & $\begin{array}{l}\text { Primärsprache, } \\
\text { 'Muttersprache' }\end{array}$ & Sekundärsprache & $\begin{array}{l}\text { prototypische } \\
\text { Fremdsprache }\end{array}$ \\
\hline Erwerbsalter & früher & später & später \\
\hline Erwerbsart & ungesteuert & $\begin{array}{l}\text { passiv: ungesteuert, } \\
\text { aktiv: teilgesteuert }\end{array}$ & i. d. R. gesteuert \\
\hline Leseverstehen & vollständig & vollständig & unvollständig \\
\hline Hörverstehen & vollständig & vollständig & unvollständig \\
\hline Schreibfertigkeit & vollständig & $\begin{array}{l}\text { vollständig, evtl. } \\
\text { weniger stilistische } \\
\text { Register } \\
\end{array}$ & unvollständig \\
\hline Sprechfertigkeit & vollständig & $\begin{array}{l}\text { weniger stilistische } \\
\text { Register }\end{array}$ & unvollständig \\
\hline $\begin{array}{l}\text { Selbsteinschätzung der } \\
\text { eigenen Kompetenz: } \\
\text { produktiv }\end{array}$ & hoch & defizitär & defizitär \\
\hline $\begin{array}{l}\text { Selbsteinschätzung der } \\
\text { eigenen Kompetenz: } \\
\text { rezeptiv }\end{array}$ & hoch & hoch & defizitär \\
\hline Verwendung & $\begin{array}{l}\text { häufiger (von der } \\
\text { Kommunikations- } \\
\text { situation abhängig) } \\
\end{array}$ & $\begin{array}{l}\text { seltener (von der } \\
\text { Kommunikations- } \\
\text { situation abhängig) } \\
\end{array}$ & $\begin{array}{l}\text { vom Kommunikations- } \\
\text { partner abhängig }\end{array}$ \\
\hline Ausgebautheit & $\mathrm{ja} / \mathrm{nein}$ & ja & $\mathrm{ja}$ \\
\hline Standardisiertheit & $\mathrm{ja} / \mathrm{nein}$ & ja & $\mathrm{ja}$ \\
\hline $\begin{array}{l}\text { kommunikative } \\
\text { Reichweite }\end{array}$ & stärker begrenzt & weniger stark begrenzt & $\begin{array}{l}\text { 'internationale' } \\
\text { Kommunikation }\end{array}$ \\
\hline Prestige & situativ abhängig & situativ abhängig & hoch \\
\hline affektive Einstellung & $\begin{array}{l}\text { Vertrautheit, Nähe, } \\
\text { Wärme, emotionale } \\
\text { Heimat }\end{array}$ & $\begin{array}{l}\text { Funktionalität, } \\
\text { Formalität, Distanz, } \\
\text { Kühle }\end{array}$ & Fremdheit \\
\hline $\begin{array}{l}\text { Symbolisierung von } \\
\text { lokaler/regionaler/nati- } \\
\text { onaler Identität }\end{array}$ & ja & nein & $\begin{array}{l}\text { fremde Identität, wenn } \\
\text { nicht lingua franca }\end{array}$ \\
\hline
\end{tabular}

Tabelle 3: Soziolinguistische Charakteristika von Primär- und Sekundärsprache ${ }^{32}$

In sprachsystematischer Perspektive beeinflussen sich Primär- und Sekundärsprache gegenseitig: Lexikalische oder idiomatische Entlehnungen, aber auch syntaktische und morphologische Interferenzen finden sich in beiden Sprachen. Soziolinguistisch bleibt von dieser Kategorisierung die Frage unberührt, ob die Sprachensituation als gesellschaftlicher Bilingualismus oder Diglossie zu betrachten sei. Auch hinsichtlich der Unterscheidung in "High" und "Low", die Ferguson als Charakteristikum diglossischer Sprachensituationen annimmt, verhält sich das Konzept neutral.

32 Der Begriff der Zweitsprache, der in diesem Zusammenhang ebenfalls von Interesse wäre, umfasst sehr unterschiedliche Konzepte (cf. Deutsch als Zweitsprache von Migranten in Deutschland, Russisch als Zweitsprache in der ehemaligen Sowjetunion). Daher wird er hier ausgeklammert. 
Die Besonderheit der Deutschschweiz liegt nun darin, dass die Sekundärsprache in mindestens zwei Varietäten Verwendung findet: dem Standarddeutschen in seiner schweizerischen und seiner deutschländischen Variante. Während die Standardvarietät Deutschlands eher mit 'Ausland' identifiziert wird, hat das Schweizerhochdeutsch, in den dafür vorgesehenen Situationen angewendet, für die meisten Deutschschweizer weniger Fremdes. Dessen ungeachtet sind aber die Unterschiede zwischen diesen beiden Varietäten so gering, dass sie oft nicht bewusst wahrgenommen und als ein Idiom 'Standarddeutsch' behandelt werden (so auch im 'muttersprachlichen' Unterricht in der Schule) - mit entsprechend widersprüchlichen Implikationen für Spracherwerb und -verwendung. Wir sind überzeugt, dass der Status des Standarddeutschen in der Deutschschweiz mit dem Konzept der Sekundärsprache zutreffender beschrieben ist als mit dem der Fremdsprache, da es z. B. nach aktiven und passiven sowie mündlichen und schriftlichen Fertigkeiten unterscheiden kann. Der Vorzug des Konzeptes der Sekundärsprache liegt vor allem darin, dass es einerseits nicht auf eine Einzelvarietät festgelegt ist, so dass eine Sekundärsprache sich durchaus in verschiedenen Varietäten realisieren kann, es andererseits aber auch keinerlei sprachpolitische Implikationen enthält. Es dürfte daher besser geeignet sein, die Debatten in der angewandten Linguistik differenziert und pragmatisch, d. h. orientiert an der verwickelten Sprachsituation in der Deutschschweiz voranzutreiben.

\section{Literaturangaben}

Ahokas, Carmela (2003): Die Förderung der deutschen Sprache durch die Schweiz. Möglichkeiten und Einschränkungen. Frankfurt am Main etc. (= Finnische Beiträge zur Germanistik 8).

Altweg, Jürg (2004): "Bitte kein Hochdeutsch! Entscheidet sich das Schicksal der Reform in der Schweiz?". Frankfurter Allgemeine Zeitung vom 17.08.2004: 37.

Ammon, Ulrich (1986): "Explikation der Begriffe 'Standardvarietät' und 'Standardsprache' auf normtheoretischer Grundlage". In: Holtus, Günter/Radtke, Edgar (eds.): Sprachlicher Substandard. Tübingen: 1-63. (= Konzepte der Sprach- und Literaturwissenschaft 36).

Ammon, Ulrich (1995): Die deutsche Sprache in Deutschland, Österreich und der Schweiz. Das Problem der nationalen Varietäten. Berlin/New York.

Barkowski, Hans (2003): "Deutsch als Zweitsprache". In: Bausch, Karl-Richard et al. (eds.): Handbuch Fremdsprachenunterricht. Tübingen/Basel: 525-529.

Baur, Arthur (1983): Was ist eigentlich Schweizerdeutsch? Winterthur.

Baur, Arthur (1990): Schweizerdeutsch - woher und wohin? Zürich.

Berthele, Raphael (2004): "Vor lauter Linguisten die Sprache nicht mehr sehen. Diglossie und Ideologie in der deutschsprachigen Schweiz". In: Christen, Helen (ed.): Dialekt, Regiolekt und Standardsprache im sozialen und zeitlichen Raum. Wien: 111-136.

Bickel, Hans (2001): "Schweizerdeutsch; kein minderwertiges Hochdeutsch! Das Deutsche als plurizentrische Sprache aus Schweizer Sicht". Babylonia 2/01: 19-22.

Bilton, Paul (1997): Die Schweizer pauschal. Frankfurt am Main. 
Böhler, Michael (1991): "Das Verhältnis der Deutschschweizer Autoren zur Schriftsprache". In: Pezold, Klaus (ed.): Geschichte der deutschsprachigen Schweizer Literatur im 20. Jahrhundert. Berlin: 309-318.

Böhme-Dürr, Karin (1994): "Der Erwerb von Standardsprache und Dialekt durch Medien." In: Burger, Harald/Häcki Buhofer, Annelies (eds.): Spracherwerb im Spannungsfeld von Dialekt und Hochsprache. Frankfurt am Main etc.: 43-55. (= Zürcher Germanistische Studien 38).

Burri, Ruth M./Geiger, Werner/Schilling, Roswita (1993): Deutsch sprechen am Schweizer Radio DRS. Bern.

Christen, Helen (1998): Dialekt im Alltag. Eine empirische Untersuchung zur lokalen Komponente heutiger schweizerdeutschen Varietäten. Tübingen (= Reihe Germanistische Linguistik 201).

Clalüna-Hopf, Monika/Plettenberg, Marilu (1988): Deutsch im Beruf. Hotellerie und Gastronomie. Bonn-Bad Godesberg.

Clyne, Michael (1997): "Multilingualism". In: Coulmas, Florian (ed.): The Handbook of Sociolinguistics. Oxford: 301-314. (= Blackwell Handbooks in Linguistics).

Ferguson, Charles A. (1959): "Diglossia". Word 15: 325-340.

Fishman, Joshua A. (1975): Soziologie der Sprache. Eine interdisziplinäre sozialwissenschaftliche Betrachtung der Sprache in der Gesellschaft. München. (= Hueber Hochschulreihe 30).

Feuz, Barbara (2001): "Dialektale Varietät als Fremdsprache unterrichten. Ein Erfahrungsbericht". Linguistik online 9, 2/01.

http.//www.linguistik-online.de/9_01/Feuz.html

Götze, Lutz/Grub, Frank Thomas/Pommerin, Gabriele (2003): "Deutsch als Fremdsprache". In: Bausch, Karl-Richard et al. (eds.): Handbuch Fremdsprachenunterricht. Tübingen/ Basel: 521-525.

Haas, Walter (1988): "Schweiz". In: Ammon, Ulrich/Dittmar, Norbert/Mattheier, Klaus J. (eds.): Sociolinguistics / Soziolinguistik. Ein internationales Handbuch zu Wissenschaft von Sprache und Gesellschaft. 2. Band. Berlin/New York: 1365-1383. (= HSK 3.2).

Haas, Walter (2004): "Die Sprachsituation der deutschen Schweiz und das Konzept der Diglossie". In: Christen, Helen (ed.): Dialekt, Regiolekt und Standardsprache im sozialen und zeitlichen Raum. Wien: 81-110.

Häcki Buhofer, Annelies et al. (1994): "Früher Hochspracherwerb in der deutschen Schweiz: Der weitgehend ungesteuerte Erwerb durch sechs- bis achtjährige Deutschschweizer Kinder". In: Burger, Harald/Häcki Buhofer, Annelies (eds.): Spracherwerb im Spannungsfeld von Dialekt und Hochsprache. Frankfurt am Main etc.: 147-198. (= Zürcher Germanistische Studien 38).

Häcki Buhofer, Annelies/Burger, Harald (1998): Wie Deutschschweizer Kinder Hochdeutsch lernen: der ungesteuerte Erwerb des gesprochenen Hochdeutschen durch Deutschschweizer Kinder zwischen sechs und acht Jahren. Stuttgart. (= Zeitschrift für Dialektologie und Linguistik. Beihefte 98).

Hägi, Sara (2000): Helvetismen im Werk Deutschschweizer Schriftstellerinnen/Schriftsteller der Gegenwart. Unveröfftl. Examensarbeit. Universität zu Köln. 
Hofer, Lorenz (1999): "Ein Wörterbuch mit nationalen Varianten des Deutschen". Sprachspiegel: 1: 7-15.

Hoffmann, Charlotte (1991): An Introduction to Bilingualism. London/New York. (= Longman linguistics library).

Hove, Ingrid (2002): Die Aussprache der Standardsprache in der deutschen Schweiz. Tübingen.

Kloss, Heinz (1976): "Abstandsprachen und Ausbausprachen". In: Göschel, Joachim/Nail, Norbert/van der Elst, Gaston (eds.): Zur Theorie des Dialekts. Wiesbaden: 301-322. (= ZDL-Beihefte N.F.16).

Kolde, Gottfried (1981): Sprachkontakte in gemischtsprachigen Städten. Vergleichende Untersuchungen über Voraussetzungen und Formen sprachlicher Interaktion verschiedensprachiger Jugendlicher in den Schweizer Städten Biel/Bienne und Fribourg/Freiburg i.Ue. Wiesbaden. (= Zeitschrift für Dialektologie und Linguistik. Beihefte 37).

Koller, Werner (1992): Deutsche in der Deutschschweiz. Aarau/Frankfurt am Main/Salzburg. (= Reihe Sprachlandschaft 10)

Kremnitz, Georg (1987): "Diglossie/Polyglossie". In: Ammon, Ulrich/Dittmar, Norbert/Mattheier, Klaus J. (eds.): Sociolinguistics / Soziolinguistik. Ein internationales Handbuch zu Wissenschaft von Sprache und Gesellschaft. 2. Band. Berlin/New York: 208218. (= HSK 3.1)

Krohn, Tim (1998): "Zum Schreiben zwischen Nord- und Schweizerdeutsch". Sprachspiegel 4: $168-172$.

Langner, Michael (2001): "Entwicklungen des Faches Deutsch als Fremdsprache und des Deutsch als Fremd- und Zweitsprache-Unterrichts in der Schweiz". In: Helbig, Gerhard et al. (eds.): Deutsch als Fremdsprache. Ein internationales Handbuch. Berlin/New York: 108-124.

Loetscher, Hugo (1986): "Für eine Literatur deutscher Ausdrucksweise: Nicht ganz unpersönliche Ausführungen". In: Löffler, Heiner (ed.): Das Deutsch der Schweizer: Zur Sprach- und Literatursituation der Schweiz. Aarau/Frankfurt am Main/Salzburg: 25-39.

Lüdi, Georges (1996): "Mehrsprachigkeit". In: Goebl, Hans et al. (eds.): Kontaktlinguistik. Ein internationales Handbuch zeitgenössischer Forschung. 1. Halbband. Berlin/New York: 233-245. (= HSK 12.1)

Maurer, Ernst (2000): Deutsch in der Schweiz. Ein Sprachkurs für Erwachsene und Jugendliche. Zug.

Moser, Milena (1998): "Coiffeur heisst auf Deutsch Frisör". Sprachspiegel 4: 173-174.

Oomen-Welke, Ingelore (2003): "Muttersprachen- und Fremdsprachenunterricht". In: Bausch, Karl-Richard et al. (eds.): Handbuch Fremdsprachenunterricht. 4. Auflage. Tübingen/ Basel: 145-151.

Polenz, Peter von (1999): Deutsche Sprachgeschichte vom Spätmittelalter bis zur Gegenwart. Band III. 19. und 20. Jahrhundert. Berlin/New York. (= De-Gruyter-Studienbuch)

Romaine, Suzanne (2000): Language in Society. An Introduction to Sociolinguistics. Second Edition. Oxford. 
Ris, Roland (1990): "Dialektologie zwischen Linguistik und Sozialpsychologie". In: Vouga, Jean-Pierre (ed.): La Suisse face à ses langues - Die Schweiz im Spiegel ihrer Sprachen. Aarau: 40-49.

Scharloth, Joachim (2003): "Zwischen Fremdsprache und nationaler Varietät. Untersuchungen zum Plurizentrizitätsbewusstsein der Deutschschweizer." TRANS. Internet-Zeitschrift für Kulturwissenschaften 15.

http://www.inst.at/trans/15Nr/06_1/ scharloth15.htm

Schläpfer, Robert/Gutzwiller, Jürg/Schmid, Beat (1991): Das Spannungsfeld zwischen Mundart und Standardsprache in der deutschen Schweiz. Spracheinstellungen junger Deutsch- und Welschschweizer. Aarau/Frankfurt am Main.

Schmidlin, Regula (1999): Wie deutschschweizer [sic!] Kinder schreiben und erzählen lernen. Textstruktur und Lexik von Kindertexten aus der Deutschschweiz und aus Deutschland. Tübingen. (= Basler Studien zur deutschen Sprache und Literatur 79)

Sieber, Peter (1990): Perspektiven einer Deutschdidaktik für die deutsche Schweiz. Aarau/Frankfurt am Main/Salzburg. (= Reihe Sprachlandschaft 8)

Sieber, Peter (1992): "Hochdeutsch in der Schweiz". Der Deutschunterricht 44 (6): 28-42.

Sieber, Peter (2001): "Das Deutsche in der Schweiz". In: Helbig, Gerhard et al. (eds.): Deutsch als Fremdsprache. Ein internationales Handbuch. 1. Halbband. Berlin/New York: 491-504. (= HSK 19.1)

Sieber, Peter/Sitta, Horst (1986): Mundart und Standardsprache als Problem der Schule. Frankfurt a. M. (= Reihe Sprachlandschaft 3)

Sieber, Peter/Sitta, Horst (1987): "Deutsch in der Schweiz". Zeitschrift für Germanistik 8: 389-401.

Sieber, Peter/Sitta, Horst (1994): "Zur Rolle der Schule beim Aufbau von Einstellungen zu Dialekt und Standardsprache". In: Burger, Harald/Häcki Buhofer, Annelies (eds.): Spracherwerb im Spannungsfeld von Dialekt und Hochsprache. Frankfurt am Main etc.: 199-241. (= Zürcher Germanistische Studien 38)

Stern, Otto (1988): "Divergence and Convergence of Dialect and Standard from the Perspective of the Language Learner. Standard language acquisition by the Swiss-German dialect speaking child". In: Auer, Peter/di Luzio, Aldo (eds.): Variation and convergence. Studies in social dialectology. Berlin/New York: 134-156.

Trudgill, Peter (2003): A Glossary of Sociolinguistics. Edinburgh.

Studer, Thomas (2001): "Deutsch in der Schweiz. Informationen zur Sprachsituation und ein Vergleich zwischen Mundart und Hochdeutsch. Erläuterungen der Tonaufnahmen auf der CD 'Grüezi mitenand', entstanden anlässlich der IDT 2001 in Luzern". In: Grüezi mitenand. Luzernerinnen und Luzerner erzählen von sich und ihrer Stadt. Eine CD-Rom zum Schweizerdeutschen. Konzept: Monika Clalüna und Bruno Frischherz. Kriens.

Studer, Thomas (2002): "Dialekte im DaF-Unterricht? Ja, aber... Konturen eines Konzepts für den Aufbau einer rezeptiven Varietätenkompetenz". Linguistik online 10, 1/02. http://www.linguistik-online.de/10_02/studer.html

Ulrich, Ammon et al. (eds.) (2004): Variantenwörterbuch der deutschen Sprache. Die Wortschatzbesonderheiten des Standarddeutschs in Deutschland, Österreich und der Schweiz sowie in Belgien, Liechtenstein, Luxemburg und Südtirol. Berlin/New York. 
Weisgerber, Bernhard (1996): "Mundart, Umgangssprache, Standard". In: Goebl, Hans et al. (eds.): Kontaktlinguistik. Ein internationales Handbuch zeitgenössischer Forschung. 1. Halbband. Berlin/New York: 258-271. (= HSK 12.1)

Werlen, Erika (1993): "Dialekt als Norm. Hochdeutsch als Abweichung. Zur Situation von Dialekt und Schule in der deutschsprachigen Schweiz". In: Klotz, Peter/Sieber, Peter (eds.): Vielerlei Deutsch. Umgang mit Sprachvarietäten in der Schule. Stuttgart etc.: 94109.

Werlen, Erika/Ernst, Karl (1994): "Dialektale und hochsprachliche Kommunikationskultur von Schulkindern. Hypothesen und Zugänge". In: Burger, Harald/Häcki Buhofer, Annelies (eds.): Spracherwerb im Spannungsfeld von Dialekt und Hochsprache. Frankfurt am Main etc.: 215-241. (= Zürcher Germanistische Studien 38)

Werlen, Iwar (1998): "Mediale Diglossie oder asymmetrische Zweisprachigkeit? Mundart und Hochsprache in der deutschen Schweiz". Babylonia 1: 22-35.

Werner, Markus (1998): Bis bald. 4. Auflage. München. 